\title{
El género Hypoxylon (Xylariales, Ascomycota) en el bosque tropical caducifolio de cuatro Áreas Naturales Protegidas de México
}

\section{The genus Hypoxylon (Xylariales, Ascomycota) in the tropical dry forest of four Protected Natural Areas from Mexico} Acta Botanica Mexicana

\author{
Pamela E. Reyes',2 (iD, Ricardo Valenzuelal (D), Tania Raymundo,3 (iD)
}

\section{Resumen:}

Antecedentes y Objetivos: El género Hypoxylon (Hypoxylaceae) tiene peritecios embebidos en un estroma oscuro o de colores brillantes, efusoaplanado, efuso-pulvinado o glomerulado, ascas cilíndricas con poro apical amiloide, ascosporas elipsoides, equiláteras o inequiláteras, marrón claro a marrón oscuro, línea germinal recta o sigmoide y perisporio generalmente dehiscente con $\mathrm{KOH}$ al 10\%. El género tiene una distribución mundial; se han descrito 115 especies con mayor diversidad en las regiones tropicales. En México se tenían registrados 36 taxones, de los cuales 20 se citan del bosque tropical caducifolio. El objetivo del presente estudio es ampliar la distribución conocida y describir las especies de Hypoxylon que crecen en el bosque tropical caducifolio de cuatro Áreas Naturales Protegidas (ANPs) de México.

Métodos: Se realizaron seis exploraciones en las siguientes ANPs de México: Reserva de la Biosfera Chamela-Cuixmala (Jalisco), Reserva de la Biosfera Isla Cozumel (Quintana Roo), Reserva de la Biosfera Sierra de Álamos-Río Cuchujaqui (Sonora) y Reserva de la Biosfera El Cielo (Tamaulipas). Los ejemplares recolectados fueron estudiados, descritos y determinados de acuerdo con las técnicas tradicionales en micología. El material está depositado en el herbario ENCB.

Resultados clave: Se determinaron 11 especies de Hypoxylon, y se describe por primera vez para México Hypoxylon jaklitschii de Jalisco, Quintana Roo y Tamaulipas. Además, se amplía la distribución conocida de $H$. anthochroum, $H$. cinnabarinum, $H$. erythrostroma, $H$. haematostroma, $H$. investiens, H. lividipigmentum, H. perforatum, H. rickii e Hypoxylon subgilvum a otros estados.

Conclusiones: Hypoxylon está bien representado en México, siendo uno de los mejor estudiados del orden Xylariales. Hypoxylon cinnabarinum, $H$. haematostroma e $\mathrm{H}$. lenormandii se encontraron en todas las ANPs del presente estudio. La Reserva de la Biosfera el Cielo es el ANP con mayor número de especies con nueve.

Palabras clave: distribución, Hypoxylaceae, nuevos registros, Sordariomycetes, taxonomía.

\section{Abstract:}

Background and Aims: The genus Hypoxylon has perithecia immersed in stromata, shiny to dark coloured, effuse to pulvinate or glomerulate; asci cylindrical with apical amyloid pore with ellipsoid, equilateral o inequilateral ascospores, brown to dark brown with germinal line right or sigmoid and perispore dehiscent with $\mathrm{KOH} \mathrm{10 \% .} \mathrm{The} \mathrm{genus} \mathrm{has} \mathrm{a} \mathrm{worldwide} \mathrm{distribution} \mathrm{and} 115$ species have been described with highest diversity in tropical regions. In Mexico, 36 species have been registered, of which 20 occur in the tropical dry forest. The objective of the present study is to expand the distribution of and describe the Hypoxylon species that grow in tropical dry forest of four Natural Protected Areas (ANPs) from Mexico.

Methods: Six explorations were made to the following Mexican Natural Protected Areas: Chamela-Cuixmala Biosphere Reserve (Jalisco), Isla Cozumel Biosphere Reserve (Quintana Roo), Sierra de Álamos-Río Cuchujaqui Biosphere Reserve (Sonora) and El Cielo Biosphere Reserve (Tamaulipas). The specimens collected were studied, described and determined according to the traditional techniques in mycology. The material is deposited in the ENCB herbarium.

Key results: Eleven species of Hypoxylon were identified, and Hypoxylon jaklitschii is decribed for the first time to Mexico from Jalisco, Quintana Roo and Tamaulipas. Additionally, the distribution of Hypoxylon anthochroum, $\mathrm{H}$. cinnabarinum, $\mathrm{H}$. erythrostroma, $\mathrm{H}$. haematostroma, $\mathrm{H}$. investiens, $\mathrm{H}$. lividipigmentum, H. perforatum, $H$. rickii and Hypoxylon subgilvum is expanded to other states.

Conclusions: Hypoxylon is well represented in Mexico, being one of the best studied of the order Xylariales. Hypoxylon cinnabarinum, H. haematostroma and $\mathrm{H}$. lenormandii were found in all the ANPs of this study. El Cielo Biosphere Reserve is with nine the Natural Protected Area with the largest number of species.

Key words: distribution, Hypoxylaceae, new records, Sordariomycetes, taxonomy.

${ }^{1}$ Instituto Politécnico Nacional, Escuela Nacional de Ciencias Biológicas, Departamento de Botánica, Laboratorio de Micología, 11340 Cd. Mx., México. ${ }^{2}$ Instituto Politécnico Nacional, Escuela Nacional de Ciencias Biológicas, Posgrado en Biociencias, 11340 Cd. Mx., México.

${ }^{3}$ Autor para la correspondencia: raymundot.tr@gmail. com; traymundoo@ipn.com
Recibido: 17 de febrero de 2020 Revisado: 17 de marzo de 2020.

Aceptado por Marie-Stéphanie Samain: 12 de junio de 2020.

Publicado Primero en línea: 29 de junio de 2020 Publicado: Acta Botanica Mexicana 127 (2020).
Citar como: Reyes, P. E., R. Valenzuela y T. Raymundo. 2020. El género Hypoxylon (Xylariales, Ascomycota) en el bosque tropical caducifolio de cuatro Áreas Naturales Protegidas de México. Acta Botanica Mexicana 127: e1680. DOI: 10.21829/abm127.2020.1680 


\section{Introducción}

El género Hypoxylon Bull. pertenece a la familia Hypoxylaceae, del orden Xylariales, subclase Xylariomycetidae, clase Sordariomycetes del phylum Ascomycota (Wendt et al., 2018). El género se caracteriza por tener peritecios embebidos en un estroma oscuro o de colores brillantes de tipo efuso-aplanado, efuso-pulvinado o glomerulado. Los peritecios están separados y presentan formas ovoides, tubulares o globosas; éstos le confieren al estroma forma glomerulada o plana. Los ostiolos son papilados o umbilicados, además presentan pigmentos estromáticos que se evidencían al agregar $\mathrm{KOH}$ al $10 \%$. Las ascas son unitunicadas, cilíndricas, estipitadas, octosporadas con poro apical amiloide y forman ascosporas elipsoides a fusoides, equiláteras o inequiláteras, de color marrón claro a marrón oscuro, con línea germinal conspicua o inconspicua, de forma recta o sigmoide, ubicada en el lado cóncavo o convexo de las mismas. El perisporio suele ser dehiscente en presencia de $\mathrm{KOH}$ al $10 \%$ y ornamentado. Las paráfisis son septadas, filiformes y raramente ensanchadas en la parte apical (Miller, 1961; Ju y Rogers, 1996). El estado asexual (tipo Nodulisporium Preuss) de Hypoxylon se encuentra ocasionalmente en sustratos naturales y también se puede obtener al realizar aislamientos en medio de cultivo (Ju y Rogers, 1996).

Los miembros del género viven como saprótrofos o patógenos débiles en la madera de árboles de clima templado y tropical (Miller, 1961) o como endófitos (quizás en simbiosis mutualista) en los tejidos internos de plantas leñosas o herbáceas (Petrini y Petrini, 1985). Hypoxylon tiene distribución mundial, pero muestra una mayor diversidad en las regiones tropicales (Ju y Rogers, 1996), aunque se desconoce si esto refleja una realidad o simplemente es el resultado de un muestreo más intensivo en dichas áreas. Actualmente se han descrito 115 especies en el género, de los continentes africano, americano, asiático y europeo (Ju y Rogers, 1996; Van der Gucht et al., 1997; Rogers et al., 2008; Hladki y Romero, 2009; Rogers y Ju, 2012; Vasilyeva et al., 2012; Cruz y Cortez, 2015; Fournier y Lechat, 2015; Fournier et al., 2015; Kuhnert et al., 2015).

En México, Hypoxylon ha sido estudiado principalmente por Felipe San Martín (Universidad Autónoma de Tamaulipas), quien menciona que es el segundo género con mayor número de especies después de Xylaria Hill ex
Schrank en los Ascomycota. Actualmente se tiene el registro de 36 especies para Hypoxylon s.s. en el país (Miller, 1961; Martin, 1969; Pérez-Silva, 1983; Medel et al., 1989, 1999; Ju y Rogers, 1996; Pérez-Silva et al., 1996; Van der Gucht et al., 1997; San Martín et al., 1999a, b, c; Medel, 2002; Esqueda et al., 2010; Salinas-Salgado et al., 2012; Raymundo et al., 2014, 2016, 2017, 2019). En el Cuadro 1 se pueden observar las 20 especies registradas para el bosque tropical caducifolio en México con los autores que las citan, entidades federativas donde se han encontrado y, en su caso, en las ANPs estudiadas. El objetivo del presente estudio es ampliar la distribución conocida y describir las especies de Hypoxylon que crecen en el bosque tropical caducifolio de cuatro ANPs de México: Reserva de la Biosfera Chamela-Cuixmala (Jalisco), Reserva de la Biosfera Isla Cozumel (Quintana Roo), Reserva de la Biosfera Sierra de Álamos-Río Cuchujaqui (Sonora) y Reserva de la Biosfera El Cielo (Tamaulipas).

\section{Materiales y Métodos}

\section{Área de estudio}

Las Áreas Naturales Protegidas (ANPs) son zonas del territorio nacional sobre las que la nación ejerce su soberanía y jurisdicción y, lo más importante, constituyen los ambientes originales que no han sido significativamente alterados por la actividad del ser humano o que requieran ser preservadas y restauradas (CONANP, 2016). Las ANPs consideradas en el presente estudio son:

1) Reserva de la Biosfera Chamela-Cuixmala (RBCC). Es una reserva natural de México en la costa del Pacífico en el estado de Jalisco. Está limitada por los paralelos $19^{\circ} 22^{\prime} 03^{\prime \prime}$ y $19^{\circ} 35^{\prime} 11^{\prime \prime} \mathrm{N}$ y los meridianos $104^{\circ} 56^{\prime} 13^{\prime \prime}$ y $105^{\circ} 03^{\prime} 25^{\prime \prime O}$. Esta área fue creada en 1993, protege 13,142 hectáreas $\left(131.42 \mathrm{~km}^{2}\right)$. El terreno es montañoso, con selva baja caducifolia o bosque tropical seco (dependiendo de la nomenclatura), colinas y llanuras aluviales. La RBCC se ubica en la costa de Jalisco, en el municipio de La Huerta, aproximadamente a $120 \mathrm{~km}$ al norte de Manzanillo entre el margen norte del río Cuitzmala y el arroyo Chamela. La región de Chamela-Cuixmala se caracteriza por su variada y abundante fauna y por mantener extensiones considerables de bosque tropical caducifolio y humedales con poca perturbación y algunas especies dominantes son cascalo- 
Cuadro 1: Especies de Hypoxylon Bull. registradas de bosque tropical caducifolio de México. Abreviaciones: RBARC (Reserva de la Biosfera ÁlamosRío Cuchujaqui), RBEC (Reserva de la Biosfera El Cielo).

\begin{tabular}{|c|c|c|}
\hline Especie & Referencia & Estado \\
\hline H. aeruginosum J.H. Mill. & San Martín et al., 1999a & Quintana Roo \\
\hline H. anomalum J.D. Rogers, Y.M. Ju \& Oses & Raymundo et al., 2017 & Sonora: RBARC \\
\hline H. anthochroum Berk. \& Broome & San Martín et al., 1999a, c & $\begin{array}{l}\text { Chiapas, Oaxaca, Quintana Roo, Sonora: } \\
\text { RBARC }\end{array}$ \\
\hline H. cinnabarinum (Henn.) Henn. & $\begin{array}{l}\text { Guzmán-Dávalos et al., 1983; Ju y } \\
\text { Rogers,1996; San Martín et al., 1999a; } \\
\text { Medel, } 2002\end{array}$ & Colima, Jalisco, Tabasco \\
\hline H. crocopeplum Berk. \& M.A. Curtis & $\begin{array}{l}\text { Ju y Rogers, 1996; San Martín et al., 1999a; } \\
\text { Medel, 2002; Salinas-Salgado et al., } 2012\end{array}$ & $\begin{array}{l}\text { Guerrero, Oaxaca, Nayarit, Quintana Roo, } \\
\text { Tamaulipas: RBEC }\end{array}$ \\
\hline H. dieckmanii Theiss. & San Martín et al., 1999a & Oaxaca, Quintana Roo, Tamaulipas: RBEC \\
\hline H. duranii J.D. Rogers & San Martín et al., 1999a & Yucatán, Quintana Roo \\
\hline H. erythrostroma J.H. Mill. & San Martín et al., 1999a & Quintana Roo \\
\hline H. fendleri Berk. ex Cooke & San Martín et al., 1999a & $\begin{array}{l}\text { Campeche, Oaxaca, Quintana Roo, } \\
\text { Tamaulipas: RBEC }\end{array}$ \\
\hline H. fuscopurpureum (Schwein.) M.A. Curtis & Salinas-Salgado et al., 2012 & Guerrero \\
\hline H. haematostroma Mont. & $\begin{array}{l}\text { Guzmán, 1983; Ju y Rogers, 1996; San } \\
\text { Martín et al., 1999a; Medel, 2002; } \\
\text { Raymundo et al., } 2014\end{array}$ & $\begin{array}{l}\text { Campeche, Chiapas, Colima, Morelos, } \\
\text { Oaxaca, Quintana Roo, Tamaulipas: } \\
\text { RBEC, Veracruz, Yucatán }\end{array}$ \\
\hline H. hypomiltum Mont. & $\begin{array}{l}\text { Guzmán, 1983; Ju y Rogers, 1996; San } \\
\text { Martín et al., 1999a }\end{array}$ & Quintana Roo, Tabasco \\
\hline H. investiens (Schwein.) M.A. Curtis & San Martín et al., 1999a & Tabasco, Tamaulipas: RBEC, Veracruz \\
\hline H. lenormandii Berk. \& M.A. Curtis & $\begin{array}{l}\text { San Martín et al., 1999a; Medel, 2002; } \\
\text { Raymundo et al., 2014, } 2017\end{array}$ & $\begin{array}{l}\text { Oaxaca, Sonora: RBARC, Yucatán, } \\
\text { Chiapas, Nuevo León, Sinaloa, Nayarit, } \\
\text { Campeche, Tamaulipas: RBEC, Quintana } \\
\text { Roo }\end{array}$ \\
\hline H. lividipigmentum F. San Martín, Y.M. Ju \& J. D. Rogers & San Martín et al., 1999a & Oaxaca, Quintana Roo \\
\hline H. macrocarpum Pouzar & San Martín et al., 1999a & $\begin{array}{l}\text { Campeche, Quintana Roo, Tamaulipas: } \\
\text { RBEC }\end{array}$ \\
\hline H. olliforme F. San Martín, P. Lavín \& Port.-Port. & San Martín et al., 1999b & Morelos \\
\hline H. placentiforme Berk. \& M.A. Curtis & San Martín et al., 1999a & $\begin{array}{l}\text { Campeche, Quintana Roo, Tamaulipas: } \\
\text { RBEC }\end{array}$ \\
\hline H. polyporus (Starbäck) Y.M. Ju \& J.D. Rogers & San Martín et al., 1999a, c & $\begin{array}{l}\text { Quintana Roo, Sonora: RBARC, } \\
\text { Tamaulipas: RBEC }\end{array}$ \\
\hline H. texcalense F. San Martín, Port.-Port. \& P. Lavín & San Martín et al., 1999b & Morelos \\
\hline
\end{tabular}

te (Caesalpinia coriaria (Jacq.) Willd.), hediondilla (C. eriostachys Benth.), botoncillos (Cordia alliodora (Ruiz \& Pav.) Oken), ramón (Brosimum alicastrum Swartz.), cuachalala- te (Amphypterigium adstringens (Schltdl.) Standl.) y nopal (Opuntia excelsa Sánchez-Mej.) (Ceballos et al., 1999; Arriaga et al., 2000). 
2) Reserva de la Biosfera Isla de Cozumel (RBIC). Es una reserva natural ubicada en el Caribe Mexicano, en el estado de Quintana Roo, la reserva cuenta con una superficie de 134,624.17 hectáreas $\left(1346.24 \mathrm{~km}^{2}\right)$. Cozumel es una isla oceánica de origen coralino, que nunca estuvo conectada al continente. Este aislamiento la convirtió en un lugar propicio para la generación de endemismos, ya que, a lo largo del tiempo geológico, sus poblaciones han evolucionado en forma independiente. Está limitada por los paralelos $20^{\circ} 36^{\prime}$ a $20^{\circ} 16^{\prime} \mathrm{N}$ y los meridianos $86^{\circ} 44^{\prime}$ a $87^{\circ} 20^{\prime} \mathrm{O}$. Sus ecosistemas son variados, existen arrecifes coralinos, pastos marinos, manglares, vegetación de dunas costeras, tasistales-saibales y en menor proporción, bosque tropical caducifolio y un sistema de humedales y lagunas costeras. Éstos destacan por su riqueza, se reconoce la existencia de más de 700 especies que se distribuyen en grupos de flora y fauna terrestre y marina. Entre las especies de plantas dominantes tenemos al huamuche (Acacia pringlei Rose), ramón (Brosimum alicastrum), palo mulato (Bursera simaruba (L.) Sarg.), palo gusano (Caesalpinia yucatanensis (Britton \& Rose) Greenm.), guarumo (Cecropia obtusifolia Bertol.), ceibillo (Ceiba aesculifolia (Kunth) Britten), uvero (Coccoloba barbadensis Jacq.), guayacán (Guaiacum sanctum L.), cucharo (Pithecellobium mangense (Jacq.) Macbride) y yaxnik (Vitex gaumeri Greenm.) (CONANP, 2016).

3) Reserva de la Biosfera Álamos-Río Cuchujaqui (RBARC). Esta reserva se ubica dentro de las provincias geográficas de la Sierra Madre Occidental y la llanura Costera del Noroeste de México, que consiste en un gradiente altitudinal de la vegetación, desde el bosque tropical caducifolio (tierras bajas de hoja caduca) hasta los bosques de la Sierra Madre de hoja perenne (bosque de pino-roble). La Sierra de Álamos corre paralela a la costa del océano Pacífico y está atravesado por numerosos barrancos profundos que han sido excavados por los ríos que desembocan en el Pacífico como el caso del río Cuchujaqui, un afluente del río Fuerte y tiene una extensión de 92,889.6 hectáreas (928.89 km²). Está limitada por los paralelos $26^{\circ} 52^{\prime} 48^{\prime \prime}$ a $27^{\circ} 09^{\prime} 00^{\prime \prime} \mathrm{N}$ y los meridianos $108^{\circ} 34^{\prime} 12^{\prime \prime}$ a $109^{\circ} 04^{\prime} 48^{\prime \prime O}$. Fue decretada como Área de Protección de Flora y Fauna Sierra de Álamos-Río Cuchujaqui en 1996 y en 2007 por la UNESCO como Reserva de la Biosfera. Esta región es conocida tanto por la confluencia entre los límites septentrionales de muchas especies tropicales como una gran diversidad geológica y de suelos. Entre las especies representativas se tienen el palo de brasil (Haematoxylon brasiletto H. Karst.), ceiba (Ceiba acuminata (S. Watson) J.N. Rose), ciprés de Moctezuma (Taxodium huegelii C. Lawson), cedro (Cedrela odorata L.), tepeguaje (Lysiloma watsonii Rose), torote (Bursera confusa Engl.) y torote (Bursera inopinata Bullock.) (Arriaga et al., 2000; SEMARNAT, 2015).

4) Reserva de la Biosfera El Cielo (RBEC). Esta área se localiza en la porción sureste del estado de Tamaulipas, abarca ambas vertientes de una parte de la Sierra Madre Oriental en lo que se conocen como Sierra de Cucharas y Sierra Chiquita y comprende a los municipios Gómez Farías, Jaumave, Llera y Ocampo. Está limitada por los paralelos 22 $55^{\circ} 30^{\prime \prime}$ y $23^{\circ} 25^{\prime} 50^{\prime \prime} \mathrm{N}$ y los meridianos $99^{\circ} 95^{\prime} 50^{\prime \prime}$ y $99^{\circ} 26^{\prime} 30^{\prime \prime O}$. Fue decretada como Reserva de la Biosfera en 1985 y protege un área de 144,530 hectáreas, $\left(1445 \mathrm{~km}^{2}\right)$. Representa una gran porción de la Sierra Madre Oriental, por fenómenos latitudinales, geológicos y climáticos se han formado una diversidad de microhábitats que albergan un gran número de especies de origen holártico y neotropical. Las partes bajas de la sierra están cubiertas principalmente por bosque tropical caducifolio en los municipios Gómez Farías y Ocampo y los elementos más comunes son: guaje blanco (Acacia coulteri Benth.), palo mulato (Bursera simaruba), palo barba de indio (Cynophalla flexuosa (L.) J.Presl), zapotillo (Casimiroa pringlei Engl.), guasima (Guazuma ulmifolia Lam.), palo blanco (Lysiloma divaricata (Jacq.) J.F. Macbr.) y ébano (Ebenopsis ebano (Berland.) Barneby \& J.W. Grimes), entre otros (HernándezSandoval, 1998; Arriaga et al., 2000).

\section{Trabajo de campo}

De 2017 a 2018 durante la temporada de lluvias se realizaron seis exploraciones a las Áreas Naturales Protegidas (ANPs): en el mes de septiembre de 2018, se recolectó en la Reserva de la Biosfera Chamela-Cuixmala (RBCC), mientras que en la Reserva de la Biosfera Isla Cozumel (RBIC) se hicieron dos exploraciones, una en enero de 2018 y la otra a principios de junio del mismo año; después, en octubre de 2018 se realizó una salida de campo a la Reserva de la Biosfera Sierra de Álamos-Río Cuchujaqui (RBARC); y finalmente, se hicieron dos recolecciones en la Reserva de la Biosfera El Cielo (RBEC) en octubre de 2017 y a finales de junio de 2018. 
El muestreo que se realizó fue libre y sin restricciones que según Angulo et al. (2006) nos da la oportunidad de recolectar una mayor diversidad de especímenes de hongos y se aplica a todo tipo de organismos. Los especímenes recolectados se describieron morfológicamente, incluyendo sus caracteres macroscópicos de importancia taxonómica, y se registraron los datos de campo tales como: fecha, localidad, tipo de vegetación, hospedero, hábito, hábitat y nombre del recolector y determinador de la especie. Además, se tomaron fotografías in situ, utilizando cámaras Nikon D5500 (Nikon, Tokio, Japón) y Nikon D7000 (Nikon, Tokio, Japón), y posteriormente, se guardaron en cajas de plástico con separadores para preservarlos y retardar su descomposición.

\section{Trabajo de laboratorio}

Adicionalmente, se hizo la revisión de los especímenes depositados en la Colección de Hongos "Dr. Gastón Guzmán Huerta" del Herbario de la Escuela Nacional de Ciencias Biológicas (ENCB) del Instituto Politécnico Nacional de las ANPs estudiadas en el presente trabajo. Los especímenes se caracterizaron macro y micromorfológicamente de acuerdo con Ju y Rogers (1996), considerándose el tamaño y forma del peritecio, ascas, ascosporas y células estériles, haciendo cortes longitudinales en preparaciones temporales, utilizando alcohol al $70 \%$ e hidróxido de potasio $(\mathrm{KOH})$ al $10 \%$ para la extracción de pigmentos estromáticos de gran valor taxonómico. El estudio morfométrico se realizó en un microscopio óptico (MO) (K-7 Zeiss, Jena, Alemania) y para el detalle de la ornamentación de las esporas se utilizó microscopía electrónica de barrido (MEB) (JSM-5800LV, Jeol, Peabody, MA, EUA). Las especies se determinaron con las claves especializadas de Ju y Rogers (1996), San Martín et al. (1999a), Rogers et al. (2008), Hladki y Romero (2009), Rogers y Ju (2012), Vasilyeva et al. (2012), Cruz y Cortez (2015), Fournier et al. (2015) y Kuhnert et al. (2015). La terminología utilizada hace referencia al diccionario de Ulloa y Hanlin (2006).

\section{Resultados}

Se determinaron 11 especies del género Hypoxylon para cuatro Áreas Naturales Protegidas en donde predomina el bosque tropical caducifolio. Hypoxylon jaklitschii Sir \& Kuhnert se cita por primera vez de México para tres de las ANPs aquí estudiadas: RBCC (Jalisco), RBIC (Quintana Roo) y RBEC
(Tamaulipas). Hypoxylon anthochroum Berk. \& Broome se registra por primera vez para Jalisco y para la Reserva de la Biosfera Chamela-Cuixmala. Hypoxylon cinnabarinum (Henn.) Henn. se reporta por primera vez para Quintana Roo de la Reserva de la Biosfera Isla Cozumel, de Sonora en la Reserva de la Biosfera Sierra de Álamos-Río Cuchujaqui y de Tamaulipas en la Reserva de la Biosfera El Cielo; adicionalmente, esta especie se había citado de Jalisco, pero no de la Reserva de la Biosfera Chamela-Cuixmala. Hypoxylon erythrostroma J.H. Mill. se registra por primera vez de Sonora de la Reserva de la Biosfera Sierra de Álamos-Río Cuchujaqui y para la Reserva de la Biosfera Isla Cozumel. Hypoxylon haematostroma Mont. se registra por primera vez para la Reserva de la Biosfera Chamela-Cuixmala (Jalisco) y Reserva de la Biosfera Isla Cozumel (Quintana Roo). Hypoxylon investiens (Schwein.) M.A. Curtis se registra por primera vez para Quintana Roo de la Reserva de la Biosfera Isla Cozumel y de Sonora para la Reserva de la Biosfera Sierra de Álamos-Río Cuchujaqui. Hypoxylon lenormandii Berk. \& M.A. Curtis se menciona por primera vez para Jalisco de la Reserva de la Biosfera Chamela-Cuixmala. Hypoxylon lividipigmentum F. San Martín, Y.M. Ju \& J.D. Rogers se registra por primera vez para Sonora de la Reserva de la Biosfera Sierra de Álamos-Río Cuchujaqui y Tamaulipas de la Reserva de la Biosfera EI Cielo; adicionalmente, se cita por primera vez de la Reserva de la Biosfera Chamela-Cuixmala (Jalisco). Hypoxylon perforatum (Schwein.) Fr. se registra por primera vez para Jalisco de la Reserva de la Biosfera Chamela-Cuixmala y Quintana Roo de la Reserva de la Biosfera Isla Cozumel. Hypoxylon rickii Y.M. Ju \& J.D. Rogers se registra por primera vez de Quintana Roo de la Reserva de la Biosfera Isla Cozumel, Sonora de la Reserva de la Biosfera Sierra de Álamos-Río Cuchujaqui y Tamaulipas de la Reserva de la Biosfera El Cielo. Hypoxylon subgilvum Berk. \& Broome se cita por primera vez para Jalisco de la Reserva de la Biosfera Chamela-Cuixmala y Quintana Roo de la Reserva de la Biosfera Isla Cozumel.

\section{Taxonomía}

Hypoxylon anthochroum Berk. \& Broome, J. Linn. Soc., Bot. 14(74): 122. 1873. TIPO: SRI LANKA. Sobre corteza, XI.1867, G. H. K. Thwaite 160 (lectotipo: K, isolectotipos: BPI, NY). Fig. 1. 


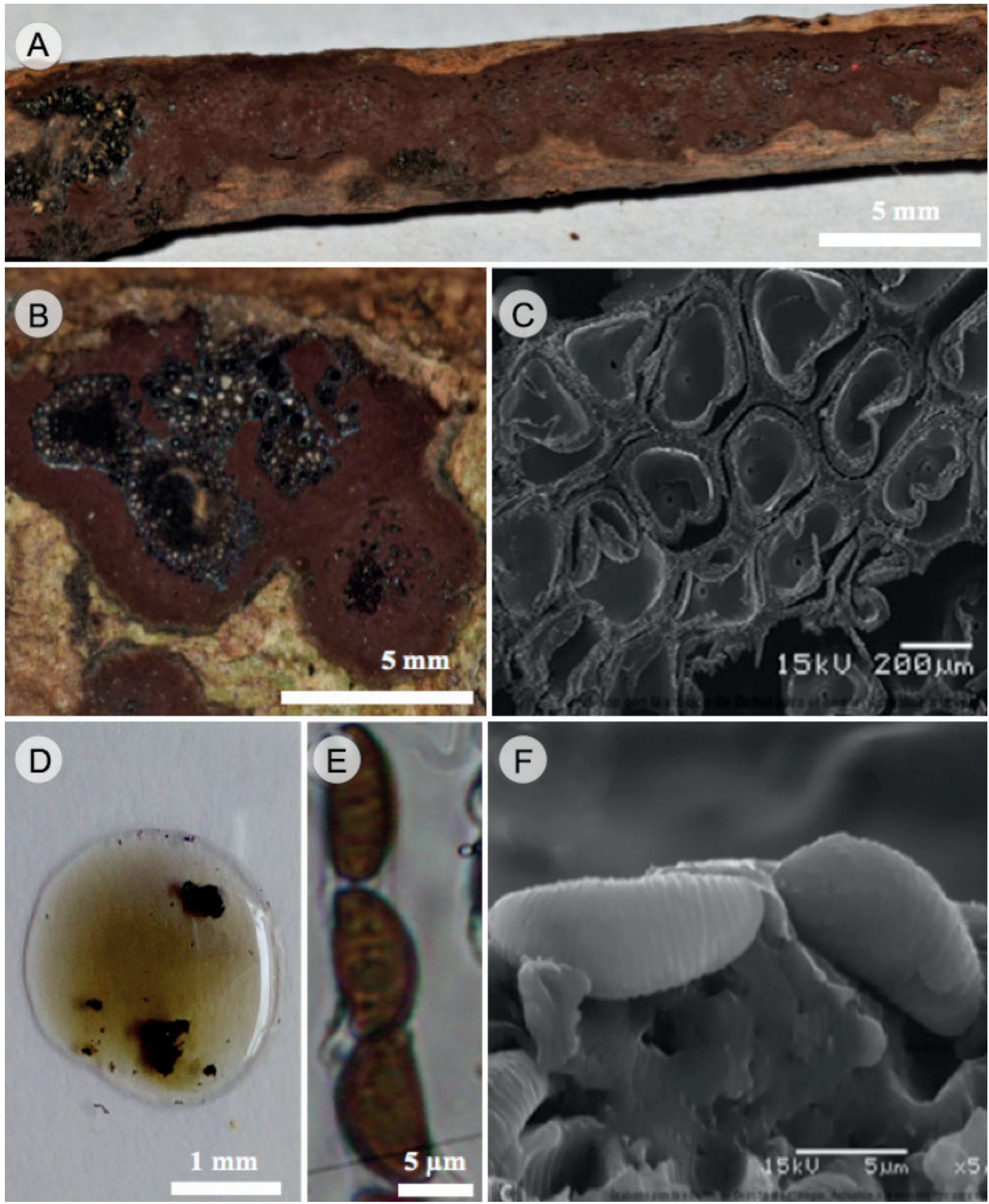

Figura 1: Hypoxylon anthochroum Berk. \& Broome. A y B. vista general de estroma efuso-pulvinado; C. peritecios ovoides vistos en MEB; D. pigmentos oliváceos en $\mathrm{KOH}$ al 10\%; E. ascas con ascosporas con perisporio dehiscente; F. ascosporas con perisporio estriado transversalmente vistas en MEB. 
= Hypoxylon murcidum Berk. \& Broome, J. Linn. Soc., Bot. 14(74): 123. 1873. TIPO: SRI LANKA. Sobre madera, s.f., G. H. K. Thwaite 1083, como Sphaeria murcida Berk. \& Broome (holotipo: K, isotipo: NY).

= Hypoxylon albostigmatosum Speg. (as 'albo-stigmatosum'), Anal. Soc. Cient. Argent. 18(6): 271. 1884. TIPO: PARAGUAY. Guarapi, sobre corteza de madera, X.1878, B. Balansa 2781 (isolectotipos: BPI, NY).

= Hypoxylon guarapiense Speg., Anal. Soc. cient. argent. 18(6): 272. 1884. TIPO: PARAGUAY. Guarapi, sobre madera, 29.VII.1881, B. Balansa 2764 (isotipos: BPI, NY).

= Hypoxylon fuscopurpureum f. corticola Starbäck, Bih. K. svenska Vetenskakad. Handl., Afd. 3 27(9): 9. 1901. TIPO: BRASIL. Mattogrosso, Rosario, sobre corteza de madera, 15.V.1894, A. M. Lindman B558 (holotipo: S).

इ Hypoxylon rubiginosum f. albostigmatosum (Speg.) Theiss., Annls mycol. 7(2): 147. 1909.

Estroma 10-20(-60) × 5-10 mm, efuso-pulvinado, marrón rojizo a marrón vináceo, endostroma con gránulos amarillos; peritecios 0.3-0.5 × 0.2-0.4 mm, ovoides, ostiolos umbilicados, desprende pigmentos oliváceos en $\mathrm{KOH}$ al $10 \%$, cambia a color ambar después de 1 minuto de contacto; ascas 97-130 × 6-9 $\mu \mathrm{m}$, cilíndricas, uniseriadas, octosporadas, amiloides; ascosporas 10-12 × 4-5 $\mu \mathrm{m}$, elipsoides, casi equiláteras, extremos redondeados, línea germinal recta a lo largo de la misma, marrón oscuro, perisporio liso en $\mathrm{MO}$, conspicuamente estriado transversalmente en MEB, dehiscente en $\mathrm{KOH}$ al $10 \%$.

Hábitat: estromas solitarios, gregarios a fusionados sobre ramas delgadas en descomposición de eudicotiledónea en bosque tropical caducifolio.

Distribución: se ha citado de Brasil (Cruz y Cortez, 2015), Hawái (Ju y Rogers, 1996; Rogers y Ju, 2012), Tailandia (Vasilyeva et al., 2012), Paraguay y Sudáfrica (Ju y Rogers, 1996). En México fue descrito por San Martín et al. (1999a) para Chiapas, Quintana Roo, Nuevo León y Oaxaca, y citado por Esqueda et al. (2010) de Sonora. Se registra por primera vez para Jalisco.
Material examinado: MÉXICO. Jalisco, municipio La Huerta, Reserva de la Biosfera Chamela-Cuixmala, Estación deBiología Chamela, IBUNAM, Vereda Búho, 19²9'40.30"N, $105^{\circ} 02^{\prime} 52.04^{\prime \prime O}, 15 . I X .2018$, P. Reyes 920 (ENCB); loc. cit., Eje Central, $19^{\circ} 29^{\prime} 58.39^{\prime \prime} \mathrm{N}, 105^{\circ} 02^{\prime} 37.97^{\prime \prime O}, 16 . I X .2018$, A. Zamora 334 (ENCB). Sonora, municipio Álamos, Reserva de la Biosfera Sierra de Álamos-Río Cuchujaqui, La Sierrita, $26^{\circ} 58^{\prime} 25.16^{\prime \prime} \mathrm{N}, 108^{\circ} 56^{\prime} 41.75^{\prime \prime O}, 27 . X .2018$, T. Raymundo 8074 (ENCB).

Notas taxonómicas: Hypoxylon anthochroum se distingue de Hypoxylon perforatum por la ausencia de discos color blanquecino rodeando los ostiolos y por el color de los pigmentos extraíbles, los cuales son ámbar en $H$. perforatum y oliváceos en $H$. anthochroum. A su vez, comparte el mismo tamaño de ascosporas con $H$. griseobrunneum (B.S. Mehrotra) J. Fourn., Kuhnert \& M. Stadler, que se caracteriza por tener pigmentos color beige.

Hypoxylon cinnabarinum (Henn.) Henn., Ann. Mus. Congo Belge, Bot. Sér. 5 2(1): 6. 1907. TIPO: BRASIL. Santa Catarina, prov. de Blumenau, sobre corteza, s.f., A. Möller s.n. (lectotipo: S). Fig. 2.

इ Nummularia cinnabarina Henn., Hedwigia 36(4): 227. 1897.

= Hypoxylon rubiginosum var. tropicum J.H. Mill., Monogr. World spec. Hypoxylon: 31. 1961. TIPO: BRASIL. Sobre corteza, s.f., J. Rick s.n. (lectotipo: GAM12781, ex BPI10529, designado por Ju y Rogers, 1996).

Estroma $12-70 \times 12-30 \mathrm{~mm}$, orbicular a efuso-pulvinado, anaranjado rojizo, endostroma con gránulos anaranjado brillantes; peritecios 0.4-0.8(-1) × 0.2-0.4 mm, tubulares, ostiolos umbilicados, desprende pigmentos anaranjados a anaranjado-rojizos en $\mathrm{KOH}$ al $10 \%$, no cambia después de 1 minuto de contacto; ascas 90-130 × 8-10 $\mu \mathrm{m}$, cilíndricas, uniseriadas, octosporadas, amiloides; ascosporas 10-12 × 5-6 $\mu \mathrm{m}$, elipsoides, equiláteras, extremos redondeados, con línea germinal recta a lo largo de la misma, marrón oscuro, perisporio liso e indehiscente en $\mathrm{KOH}$ al $10 \%$ en $\mathrm{MO}$ y en MEB. 

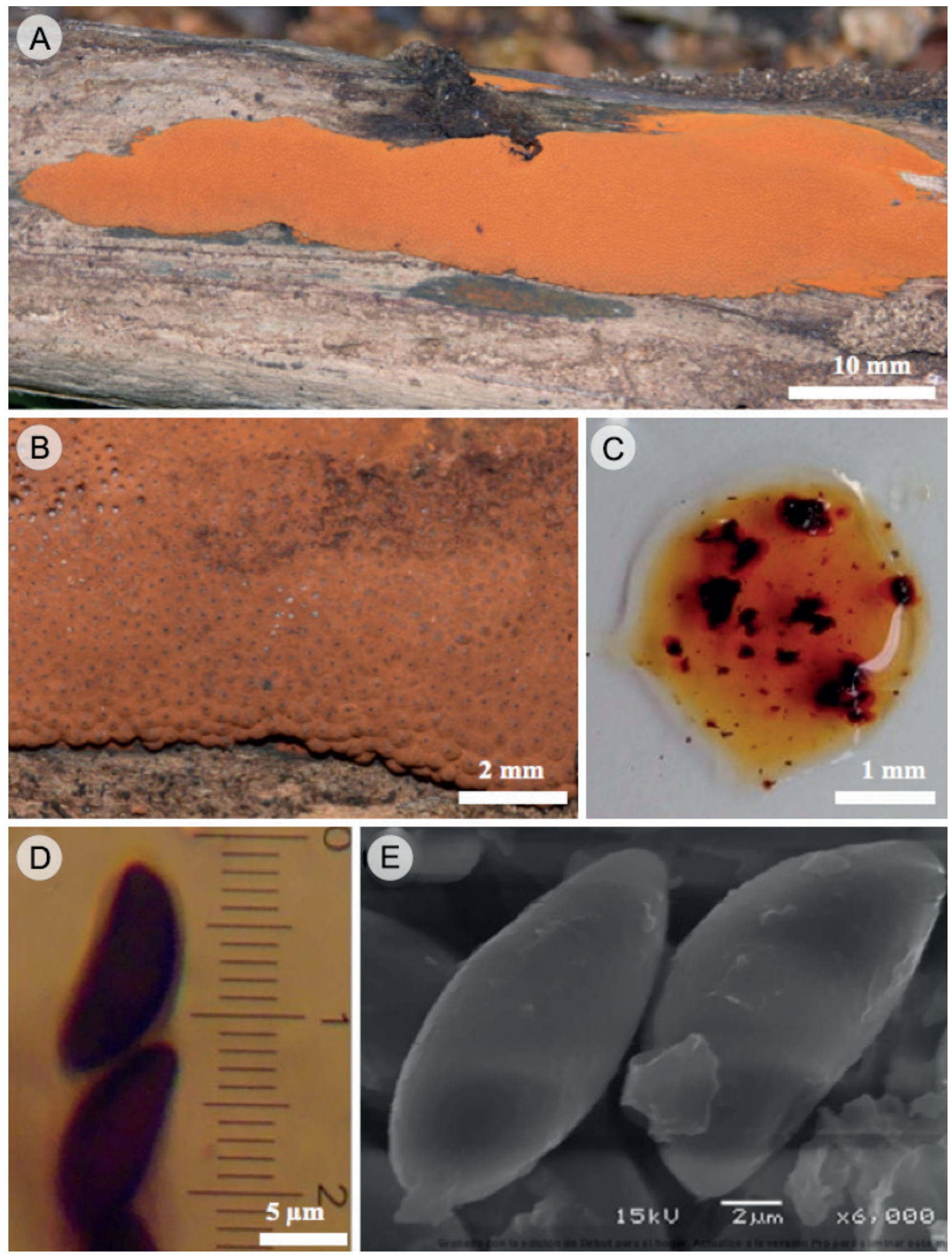

Figura 2: Hypoxylon cinnabarinum (Henn.) Henn. A y B. vista general del estroma efuso-pulvinado; C. pigmentos anaranjados en $\mathrm{KOH}$ al $10 \%$; D. ascosporas con perisporio liso e indehiscente vistas en MO; E. ascosporas lisas vistas en MEB. 
Hábitat: estromas solitarios a fusionados sobre madera en descomposición de bosque tropical caducifolio.

Distribución: se ha citado de las Antillas francesas (Fournier et al., 2015), Hawái (Rogers y Ju, 2012), Papúa Nueva Guinea (Van der Gucht et al., 1997), Nueva Zelanda, Taiwán y Venezuela (Ju y Rogers, 1996). En México fue citado de Colima (Medel, 2002), Jalisco (Guzmán-Dávalos et al., 1983) y Tabasco (Ju y Rogers, 1996). Esta especie se registra por primera vez para Quintana Roo, Sonora y Tamaulipas.

Material examinado: MÉXICO. Jalisco, municipio La Huerta, Reserva de la Biosfera Chamela-Cuixmala, Estación de Biología Chamela, IBUNAM, camino Antiguo Sur, $19^{\circ} 29^{\prime} 45.3^{\prime \prime} \mathrm{N}, 105^{\circ} 02^{\prime} 44.84^{\prime \prime O}, 22 . I X .2012$, R. Valenzuela 13950 (ENCB); loc. cit., Vereda Búho, 19²9'40.30"N, 10502'52.04"O, 15.IX.2018, P. Reyes 918 (ENCB). Quintana Roo, municipio Cozumel, Reserva de la Biosfera Isla Cozumel, Zona Arqueológica San Gervasio, 20²9'58.02' N, $86^{\circ} 50^{\prime} 37.88^{\prime \prime}$ O, 19.I.2018, R. Valenzuela 17944 (ENCB); loc. cit., Vereda de la Fundación Parques y Museos de Cozumel, 20²8'59.19"N, 8657'21.80"O, 20.I.2018, R. Valenzuela 17294 (ENCB). Sonora, municipio Álamos, Reserva de la Biosfera Sierra de Álamos-Río Cuchujaqui, El Cajón, $27^{\circ} 01^{\prime} 41.58^{\prime \prime} \mathrm{N}, 108^{\circ} 45^{\prime} 22.76^{\prime \prime} \mathrm{O}, 14 . X .2013$, D. Castro-Bustos 134 (ENCB); loc. cit., E. Escudero-Leyva 234 (ENCB); loc. cit., La Sierrita, $26^{\circ} 58^{\prime} 25.16^{\prime \prime} \mathrm{N}, 108^{\circ} 56^{\prime} 41.75^{\prime \prime} \mathrm{O}, 15 . X .2013$, R. Valenzuela 15255 (ENCB), 16966 (ENCB); loc. cit., 9.X.2014, T. Raymundo 6521 (ENCB); loc. cit., Los Cantiles, $19^{\circ} 29^{\prime} 52.09^{\prime \prime} \mathrm{N}, 105^{\circ} 2^{\prime} 37.69^{\prime \prime O}$ 9.10.2014, T. Raymundo 5513 (ENCB); loc. cit., R. Valenzuela 15886 (ENCB). Tamaulipas, municipio Gómez Farías, Reserva de la Biosfera El Cielo, La Florida, 22 ${ }^{\circ} 59^{\prime} 22^{\prime \prime} \mathrm{N}, 99^{\circ} 08^{\prime} 35^{\prime \prime} \mathrm{O}, 26 . \mathrm{VI} .2018$, P. Reyes

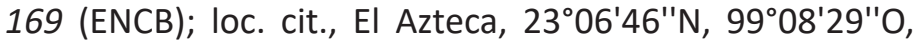
27.VI.2018, P. Reyes 175 (ENCB).

Notas taxonómicas: Hypoxylon cinnabarinum es parte del complejo de especies con estromas anaranjados a anaranjado-rojizos y pigmentos disueltos en $\mathrm{KOH}$ anaranjado-rojizos, tiene gran afinidad con $\mathrm{H}$. haematostroma, $\mathrm{H}$. fendleri Berk. ex Cooke e H. rickii. Sin embargo, los tamaños de las ascosporas son las que separan a las especies: en el caso de $H$. haematostroma son más grandes, 13.5-18(-19) $\times 7-8(-8.5) \mu \mathrm{m}$, mientras que en $\mathrm{H}$. fendleri son ligeramente más pequeñas, (8-)9-12 × 4-5.5 $\mu \mathrm{m}$, pero con el estroma rojo vináceo, y finalmente las ascosporas de $H$. rickii son más pequeñas, $6.5-8 \times 3-4 \mu \mathrm{m}$, y ornamentadas con estrías transversales, mientras que en $H$. cinnabarinum son lisas.

Hypoxylon erythrostroma J.H. Mill., Mycologia 25(4): 323. 1933. TIPO: ESTADOS UNIDOS DE AMÉRICA. Florida, Daytona, Hypoxylon no. 2, ¿̇sobre madera descortezada y ennegrecida de Ulmus?, I.1898, R. Thaxter s.n. (isotipo: GAM2374). Fig. 3.

= Hypoxylon gillesii J.D. Rogers \& Cand., Mycotaxon 15: 507. 1982. TIPO: MADAGASCAR. La Mandraka, sobre corteza, III.1981, G. Gilles s.n., Candoussau MAD 15 (holotipo: WSP 66785 , isotipo: en colección de Candoussau).

Estroma 6-30 × 4-10 mm, efuso-pulvinado, marrón a sepia grisáceo, endostroma con gránulos anaranjados a anaranjado rojizos; peritecios 0.3-0.4 mm de diámetro, inconspicuos, esféricos, ostiolos umbilicados, desprende pigmentos anaranjado amarillentos a anaranjado rojizos en $\mathrm{KOH}$ al $10 \%$, cambia a siena después de 5 minutos de contacto; ascas 90-150 × 5-7 $\mu \mathrm{m}$, cilíndricas, uniseriadas, octosporadas, amiloides; ascosporas 7.5-9 × 3-4 $\mu \mathrm{m}$, elipsoides, inequiláteras, extremos redondeados, línea germinal sigmoide, menos de la longitud total de la misma, marrón oscuro, perisporio liso en $\mathrm{MO}$, conspicuamente estriado transversalmente en MEB y dehiscente en $\mathrm{KOH}$ al $10 \%$.

Hábitat: estromas solitarios a gregarios, separados sobre madera en descomposición de eudicotiledónea en bosque tropical caducifolio.

Distribución: se ha citado de las Antillas francesas (Fournier et al., 2015), Papúa Nueva Guinea (Van der Gucht et al., 1997), Hawái (Rogers y Ju, 2012), Madagascar y Puerto Rico (Ju y Rogers, 1996). En México se ha citado de Chiapas, Quintana Roo, Tamaulipas y Veracruz (San Martín et al., 1999a). Se registra por primera vez de Sonora en la Reserva de la Biosfera Sierra de Álamos-Río Cuchujaqui y para la Reserva de la Biosfera Isla Cozumel (Quintana Roo). 

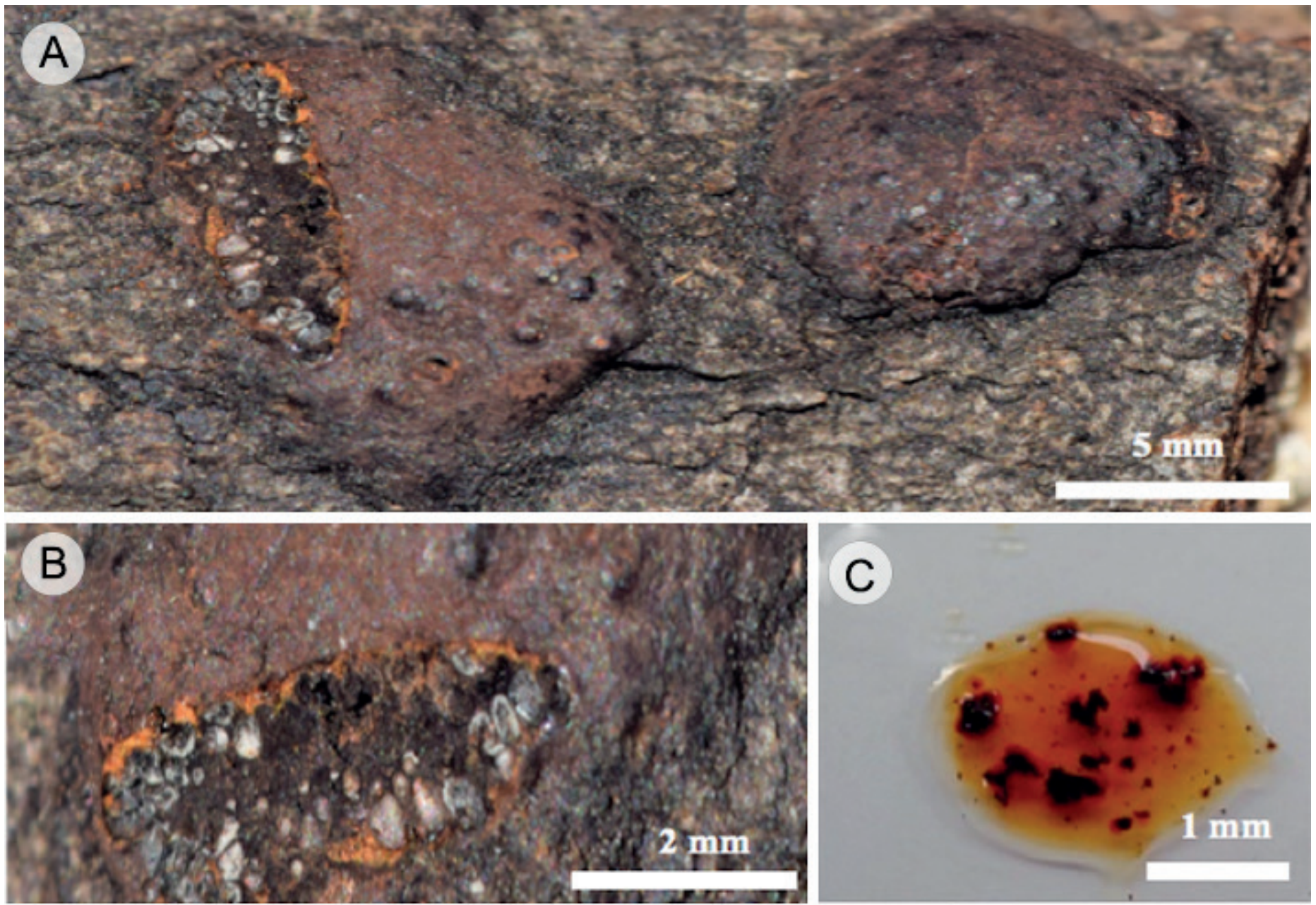

\section{C}
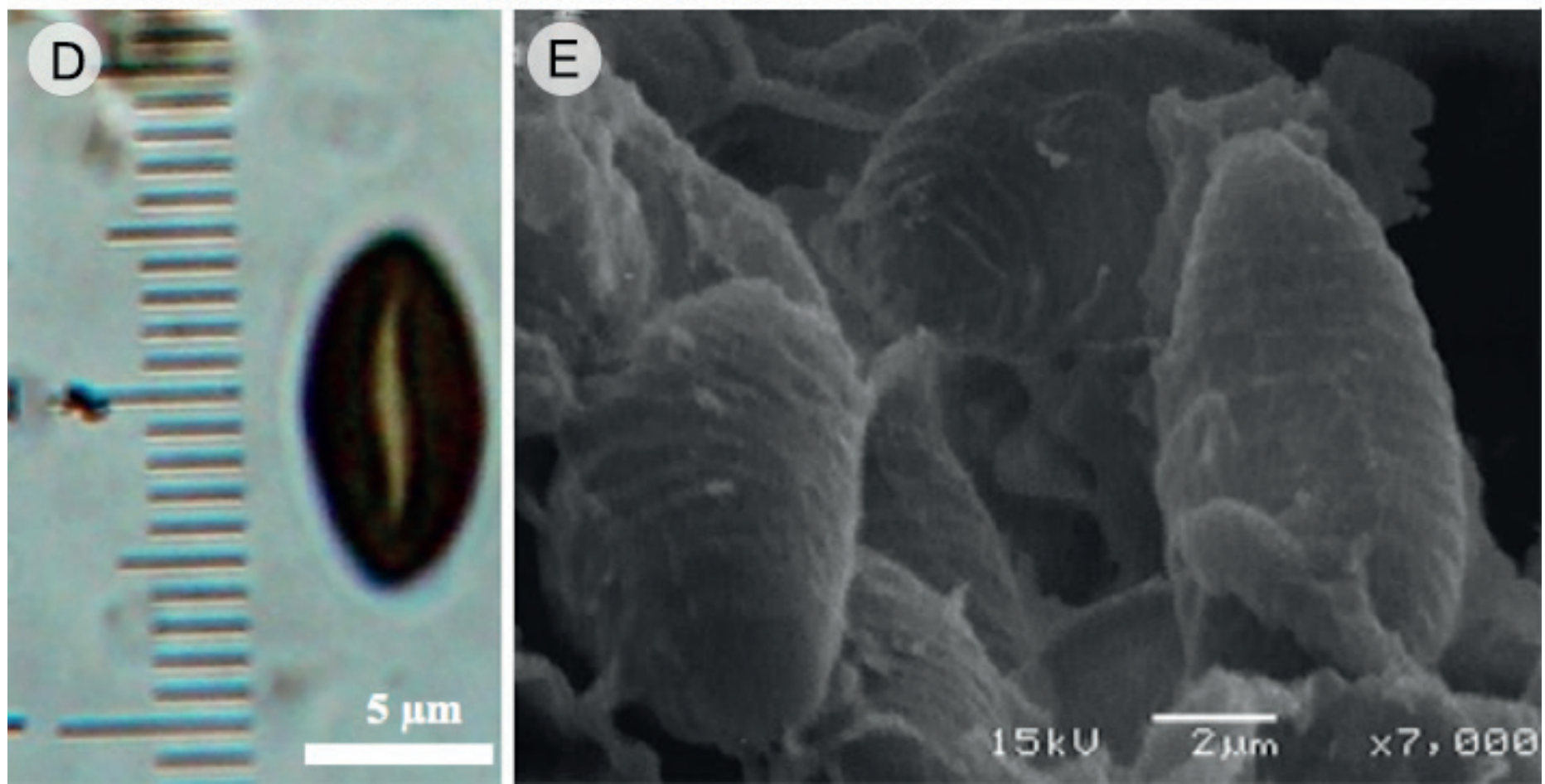

Figura 3: Hypoxylon erythrostroma J.H. Mill. A y B. vista general del estroma efuso-pulvinado con gránulos endostromatales anaranjados en corte transversal; C. pigmentos estromáticos anaranjados en $\mathrm{KOH}$ al 10\%; D. ascospora con línea germinal sigmoide vista en $\mathrm{MO}$; $\mathrm{E}$. ascosporas con perisporio estriado transversalmente vistas en MEB. 
Material examinado: MÉXICO. Quintana Roo, municipio Cozumel, Reserva de la Biosfera Isla Cozumel, Vereda de la Fundación Parques y Museos de Cozumel, 20²8'59.19"N, 8657'21.80"O, 20.I.2018, R. Valenzuela 17971 (ENCB). Sonora, municipio Álamos, Reserva de la Biosfera Sierra de Álamos-Río Cuchujaqui, Los Cantiles, 19²9'52.09"N, $105^{\circ} 2$ '37.69"O, 9.X.2014, T. Raymundo 6520 (ENCB). Tamaulipas, municipio Gómez Farías, Reserva de la Biosfera El Cielo, La Florida, 2259'22"'N, 9908'35'O, 26.VI.2018, A. Cobos-Villagrán 1945 (ENCB).

Notas taxonómicas: Hypoxylon erythrostroma se caracteriza por tener estromas de color sepia grisáceo, endostroma con gránulos anaranjados y desprende pigmentos anaranjados, mismos que son observados en $H$. howeanum Peck, pero se distingue el primero por distribuirse en bosque tropical y el último en bosques únicamente templados (Ju y Rogers, 1996; San Martín et al., 1999a).

Hypoxylon haematostroma Mont., in Sagra, Annls Sci. Nat., Bot., sér. 2 17: 124. 1842. TIPO: CUBA. Sobre madera, s.f., R. de la Sagra s.n. (holotipo: BPI55132, isotipo: K). Fig. 4.

= Hypoxylon vividum Berk. \& Broome, J. Linn. Soc., Bot. 14(74): 122. 1873. TIPO: SRI LANKA. Sobre madera, s.f., G. H. K. Thwaite 1086, como Sphaeria vivida B. \& B. (holotipo: K). = Hypoxylon haematites Lév. ex Cooke, Grevillea 11(60): 133. 1883. TIPO: ISLAS MARQUESAS. Sobre madera, s.f., Nukuhiva 8698, como Sphaeria haematites Lév. (holotipo: K). = Hypoxylon veracrucis Berk. \& Cooke, Grevillea 11(60): 129. 1883. TIPO: MEXICO. Veracruz, sobre madera, X.1854, Salle s.n. (holotipo: $\mathrm{K}$, isotipo: $\mathrm{FH}$, sheet 7413 of Patouillard Herb.).

= Hypoxylon lucidum Ellis \& Everh., Bull. Lab. Nat. Hist. lowa State Univ. 4: 72. 1896. TIPO: NICARAGUA. Sobre madera, s.f., C. L. Smith s.n. (holotipo: BPI).

= Hypoxylon stjanianum Ferd. \& Winge, Bot. Tidsskr. 29: 14 . 1908. TIPO: ESTADOS UNIDOS DE AMÉRICA. Islas Virgenes, St. John, Caroline, sobre corteza, 26.VII.1906, C. Raunkiaer 1729 (holotipo: C, isotipos: BPI6329, ex C.E. Chardon Herb.).
Estroma 15-70 × 10-30 mm, efuso-aplanado a efusopulvinado, anaranjado brillante, endostroma con gránulos anaranjados; peritecios 1.2-1.8 $\times 0.2-0.3 \mathrm{~mm}$, tubulares, ostiolos umbilicados, desprende pigmentos anaranjado escarlata en $\mathrm{KOH}$ al 10\%, no cambia después de 1 minuto de contacto; ascas 150-220 × 5-9 $\mu \mathrm{m}$, cilíndricas, uniseriadas, octosporadas, amiloides; ascosporas 13-17(-19) $\times$ 7-8 $\mu \mathrm{m}$, elipsoides, inequiláteras, extremos redondeados, línea germinal recta, marrón oscuro, perisporio liso en MO y MEB y dehiscente en $\mathrm{KOH}$ al $10 \%$.

Hábitat: estromas solitarios sobre madera en descomposición de bosque tropical caducifolio.

Distribución: esta especie de amplia distribución se ha citado de las Antillas francesas (Fournier et al., 2015), Hawái (Rogers y Ju, 2012), Papúa Nueva Guinea (Van der Gucht et al., 1997), Costa Rica, Cuba, Honduras, Indonesia, Nicaragua, Filipinas, Sri Lanka, Taiwán y Venezuela (Ju y Rogers, 1996). En México se ha citado de Campeche, Chiapas, Jalisco, Morelos, Oaxaca, Quintana Roo, Sonora, Tamaulipas, Veracruz y Yucatán (Guzmán, 1983; Ju y Rogers, 1996; San Martín et al., 1999a; Medel, 2002; Esqueda et al., 2010; Sánchez-Jácome y Guzmán-Dávalos, 2011; Raymundo et al., 2014). Se registra por primera vez para la Reserva de la Biosfera Chamela-Cuixmala (Jalisco) y Reserva de la Biosfera Isla Cozumel (Quintana Roo).

Material examinado: MÉXICO. Jalisco, municipio La Huerta, Reserva de la Biosfera Chamela-Cuixmala, Estación de Biología Chamela, IBUNAM, camino Antiguo Sur, $19^{\circ} 29^{\prime} 45.3^{\prime \prime} \mathrm{N}, 105^{\circ} 02^{\prime} 44.84^{\prime \prime O}, 16 . I X .2011$, T. Raymundo 3993 (ENCB); loc. cit., R. Valenzuela 13950 (ENCB); loc. cit., Eje Central, $19^{\circ} 29^{\prime} 58.39^{\prime \prime} \mathrm{N}, 105^{\circ} 02^{\prime} 37.97^{\prime \prime O}, 22 . I X .2012$, T. Raymundo 4441 (ENCB); loc. cit., 16.IX.2018, A. Zamora 323 (ENCB); loc. cit., Vereda Búho, 19²9'40.30"N, 10502'52.04"O, 17.IX.2011, T. Raymundo 4045 (ENCB); loc. cit., 15.IX.2018, P. Reyes 924 (ENCB); loc. cit., A. Zamora 321 (ENCB). Quintana Roo, municipio Cozumel, Reserva de la Biosfera Isla Cozumel, Coba, 20²9'41.05"N, 8657'33.75"O, 6.XI.1981, G. Guzmán 20730 (ENCB); loc. cit., Zona Arqueológica San Gervasio, 20²9'58.02"N, 

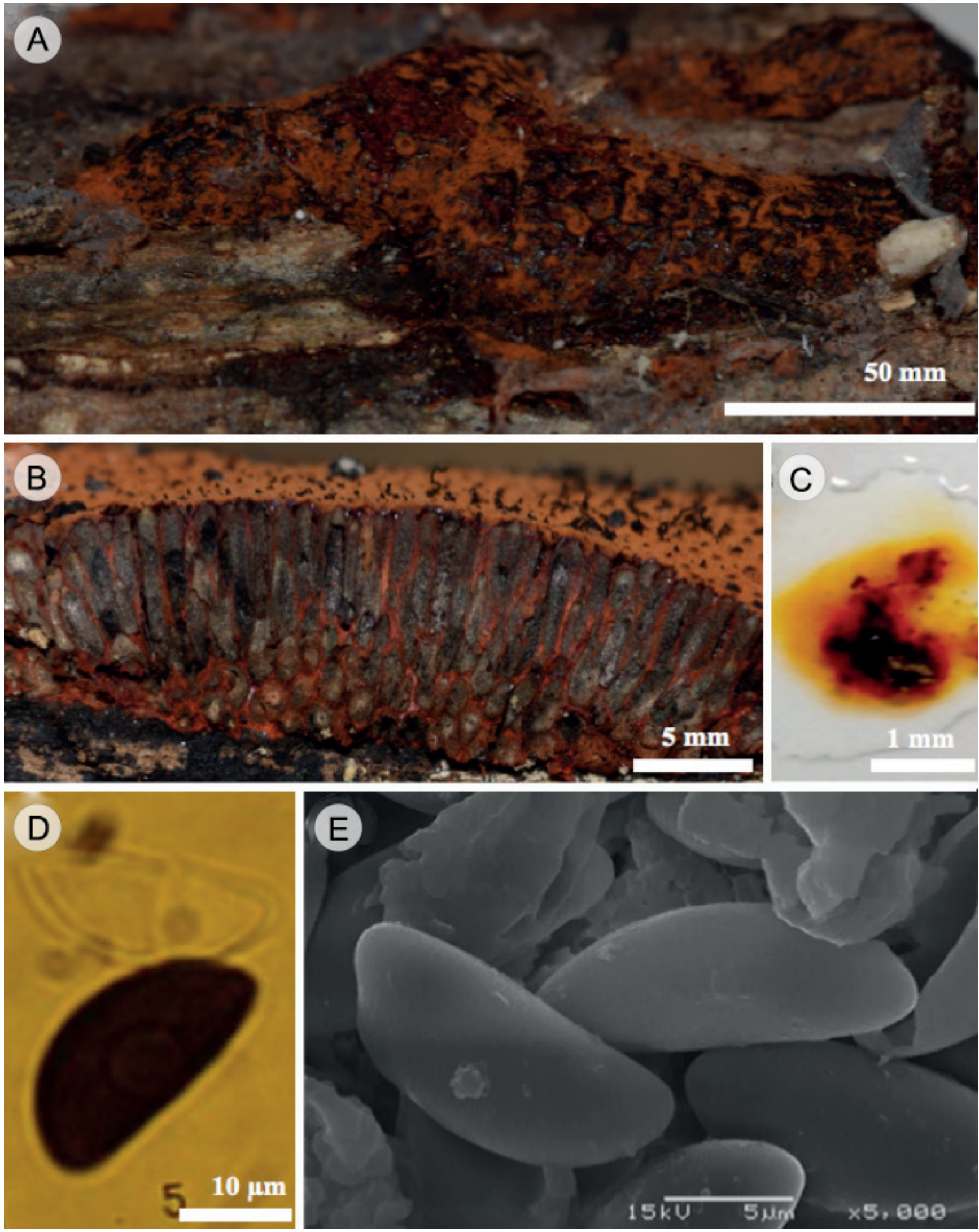

$\times 5,000$

Figura 4: Hypoxylon haematostroma Mont. A. vista general del estroma esfuso aplanado; B. corte transversal del estroma mostrando los peritecios tubulares; C. pigmentos anaranjado escarlata en $\mathrm{KOH}$ al 10\%; D. ascosporas con perisporio dehiscente vistas en $\mathrm{MO}$; E. ascosporas con perisporio liso vistas en MEB. 
8650'37.88'O, 19.I.2018, R. Valenzuela 17977 (ENCB), 17994 (ENCB); loc. cit., vereda de la Fundación de Parques y Museos, 20²8'59.19' N, 8657'21.80'O, 20.I.2018, T. Raymundo 7294 (ENCB), 7325 (ENCB). Sonora, municipio Álamos, Reserva de la Biosfera Sierra de Álamos-Río Cuchujaqui, El Cajón, $27^{\circ} 01^{\prime} 41.58^{\prime \prime} \mathrm{N}, 108^{\circ} 45^{\prime} 22.76 " \mathrm{O}, 14 . X .2013$, D. Castro-Bustos 145 (ENCB); loc. cit., T. Raymundo 4813 (ENCB); loc. cit., La Sierrita, 2658' $25.16^{\prime \prime} \mathrm{N}, 108^{\circ} 56^{\prime} 41.75^{\prime \prime O}$, 8.X.2014, T. Raymundo 5476 (ENCB), 5484 (ENCB); loc. cit., R. Valenzuela 15863 (ENCB), 15886 (ENCB), 15928 (ENCB). Tamaulipas, municipio Gómez Farías, Reserva de la Biosfera El Cielo, El Azteca, 2306'46"N, 9908'29"O, 27.VI.2018, P. Reyes 167 (ENCB).

Notas taxonómicas: Hypoxylon haematostroma es la especie característica del grupo con estroma efuso, color anaranjado y pigmentos anaranjado-rojizos. Se distingue del resto de las especies afines como $H$. cinnabarinum, $H$. fendleri e $H$. rickii, porque presenta las ascosporas más grandes que las mencionadas anteriormente.

Hypoxylon investiens (Schwein.) M.A. Curtis, Geol. Nat. Hist. Surv. N. Carol., Pt 3: 140. 1867. Fig. 5.

इ Sphaeria investiens Schwein., Trans. Am. phil. Soc., New Series 4(2): 193. 1832. TIPO: ESTADOS UNIDOS DE AMÉRICA. Pennsylvania, Northampton County, Bethlehem, sobre madera descortezada, s.f., L.D. Schweinitz 1210 (holotipo: PH). Carolina del Norte, Salem, s.f., Collins 35 (isotipo: PH). = Hypoxylon concurrens Berk. \& M.A. Curtis, in Berkeley, Grevillea 4(31): 93. 1876. TIPO: ESTADOS UNIDOS DE AMÉRICA. South Carolina, Society Hill, sobre madera descortezada, s.f., H.W. Ravenel s.n., como Sphaeria concurrens B. \& C. (holotipo: K).

= Rosellinia bakeri Ellis, Torreya 5: 87. 1905. TIPO: NICARAGUA. Chinandega, sobre madera de Urera, XII.1903, C. F. Baker 3990 (holotipo: NY00830578). = Hypoxylon investiens f. bakeri (Ellis) J.H. Mill. ex Dennis, Kew Bull. 15: 450. 1962.

= Hypoxylon microsporum A. Pande (as 'microspora'), Bull. bot. Surv. India 15: 8. 1973. TIPO: INDIA. Poona, Purandar, sobre ramas descortezadas de Eugenia, 11.III.1971, D. N. Mhaskar s.n. (holotipo: AMH1530).
= Hypoxylon investiens var. magnisporum Hladki \& A.I. Romero, Darwiniana 47(2): 283. 2009. TIPO: ARGENTINA. Buenos Aires, La Plata, Punta Lara, sobre trozo de leño semipodrido, 5.V.1971, Gómez del Busto s.n. (holotipo: BAFC51526).

Estroma 10-90 × 7-40 mm, efuso-aplanado, marrón vináceo oscuro, endostroma marrón oscuro; peritecios 0.6$0.8 \times 0.2-0.4 \mathrm{~mm}$, ovoides, ostiolos umbilicados inconspicuos, desprende pigmentos oliváceos en $\mathrm{KOH}$ al $10 \%$, no cambia después de 1 minuto de contacto; ascas 90-130 $\times$ 4-5 $\mu \mathrm{m}$, cilíndricas, uniseriadas, octosporadas, amiloides; ascosporas 7-8 $\times$ 3.5-4 $\mu \mathrm{m}$, elipsoides, equiláteras, extremos redondeados, línea germinal recta, marrón claro, perisporio liso en $\mathrm{MO}$ y $\mathrm{MEB}$, indeshiscente en $\mathrm{KOH}$ al $10 \%$.

Hábitat: estromas solitarios a gregarios sobre madera en descomposición de bosque tropical caducifolio.

Distribución: se ha citado de las Antillas francesas (Fournier et al., 2015), Hawái (Rogers y Ju, 2012), Papúa Nueva Guinea (Van der Gucht et al., 1997), Cuba, Guayana Francesa y Estados Unidos de América (Ju y Rogers, 1996). En México se ha citado de Tabasco, Tamaulipas y Veracruz (Ávalos-Lázaro et al., 2018; San Martín et al., 1999a). Se registra por primera vez para Quintana Roo y Sonora.

Material examinado: MÉXICO. Quintana Roo, municipio Cozumel, Reserva de la Biosfera Isla Cozumel, Zona Arqueológica San Gervasio, 20²9'58.02"N, 8650'37.88"O, 20.I.2018, P. Reyes 148 (ENCB); loc. cit., 17.VI.2018, R. Valenzuela 18420 (ENCB); loc. cit., vereda de la Fundación Parques y Museos de Cozumel, 20²8'59.19"N, 8657'21.80"O, 20.1.2018, R. Valenzuela 17959 (ENCB). Sonora, municipio Álamos, Reserva de la Biosfera Sierra de Álamos-Río Cuchujaqui, La Sierrita, 265' $25.16^{\prime \prime} \mathrm{N}, 108^{\circ} 56^{\prime} 41.75^{\prime \prime O}$, 8.X.2014, T. Raymundo 5559 (ENCB). Tamaulipas, municipio Gómez Farías, Reserva de la Biosfera El Cielo, El Azteca, $23^{\circ} 06^{\prime} 46^{\prime \prime} \mathrm{N}, 99^{\circ} 08^{\prime} 29^{\prime \prime} \mathrm{O}, 27 . \mathrm{VI} .2018$, P. Reyes 173 (ENCB).

Notas taxonómicas: Hypoxylon investiens es una especie de amplia distribución en climas tropicales y templados. Se caracteriza por tener un estroma efuso-aplanado, 

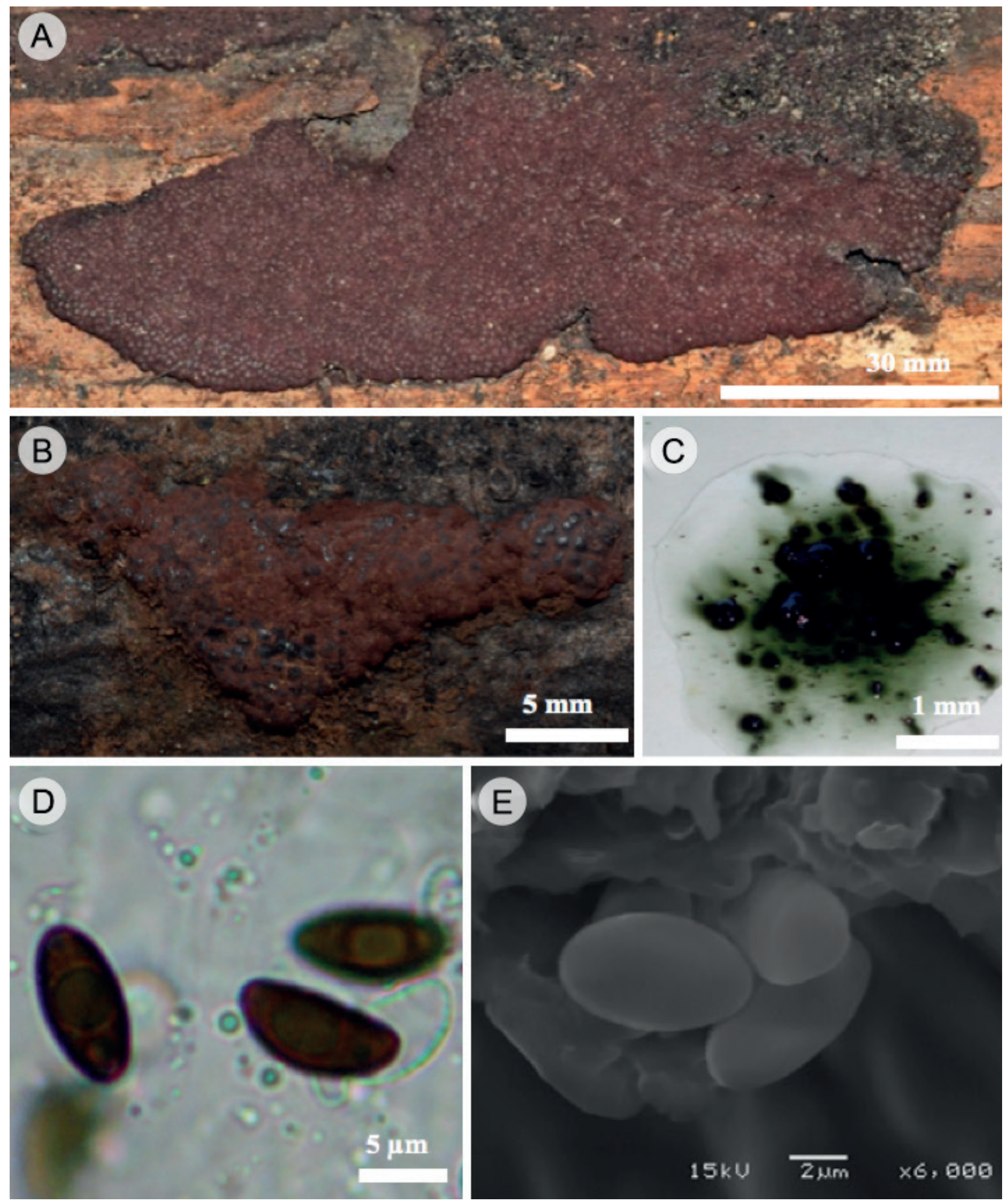

Figura 5: Hypoxylon investiens (Schwein.) M.A. Curtis. A y B. vista general del estroma efuso-aplanado; C. pigmentos oliváceos en KOH al 10\%; D. ascosporas con perisporio indehiscente en $\mathrm{KOH}$ al 10\%; E. ascosporas con perisporio liso vistas en MEB. 
color vináceo oscuro con peritecios notablemente conspicuos, pigmentos oliváceos y su tamaño de ascosporas. Recientemente Fournier et al. (2015) describieron una especie afín a $H$. investiens a la que nombraron $H$. dussii J. Fourn. \& Lechat, que se caracteriza por tener un estroma y pigmentos similares a $H$. investiens, pero con ascosporas mas grandes $(9-10 \times 4-5 \mu \mathrm{m})$.

Hypoxylon jaklitschii Sir \& Kuhnert, Fungal Diversity 71: 173. 2015. TIPO: SRI LANKA. Central Province, Sigiriya, sobre madera de árbol de hoja ancha, 16.I.2013, W. Jaklitsch JF13037 (holotipo: MUCL, ex-type cultures CBS138916 \& MUCL55383; GenBank Acc. No: ITS-KM610290, ß-tubulin-KM610304). Fig. 6.

Estroma $10-70 \times 10-40 \mathrm{~mm}$, glomerulado a efusopulvinado, sepia, marrón oscuro a negro, endostroma granulos marrón anaranjado a marrón oscuro; peritecios 0.3-0.5 mm de diámetro, conspicuos, esféricos, ostiolos umbilicados, desprende pigmentos ocres en $\mathrm{KOH}$ al $10 \%$, no cambia después de 1 minuto de contacto; ascas 120-170 $\times$ 5-7 $\mu \mathrm{m}$, cilíndricas, uniseriadas, octosporadas, amiloides; ascosporas 9.5-11.5 × 4-5.5 $\mu \mathrm{m}$, elipsoides, inequiláteras, línea germinal ligeramente sigmoide a lo largo de la misma en el lado convexo, marrón oscuro, perisporio liso en $\mathrm{MO}$, conspicuamente estriado transversamente en MEB, dehiscente en $\mathrm{KOH}$ al $10 \%$.

Hábitat: estromas gregarios y fusionados sobre madera en descomposición de Acacia sp. en bosque tropical caducifolio.

Distribución: se ha citado de Sri Lanka (Kuhnert et al., 2015). Nuevo registro para México.

Material examinado: MÉXICO. Jalisco, municipio La Huerta, Reserva de la Biosfera Chamela-Cuixmala, Estación de Biología Chamela, IBUNAM, camino Antiguo Sur, $19^{\circ} 29^{\prime} 45.3^{\prime \prime N}, 105^{\circ} 02^{\prime} 44.84^{\prime \prime O}$, 16.IX.2012, T. Raymundo 4416 (ENCB); loc. cit., R. Valenzuela 14473 (ENCB). Quintana Roo, municipio Cozumel, Reserva de la Biosfera Isla Cozumel, Zona Arqueológica de San Gervasio, 20²9'58.02" N, 8650'37.88"O, 21.I.2018, R. Valenzuela 17982 (ENCB).
Tamaulipas, municipio Gómez Farías, Reserva de la Biosfera El Cielo, El Nacimiento, 2241'30.18' N, 99²'50.50"O, 12.VI.1983, G. Rodríguez 1769 (ENCB).

Notas taxonómicas: Hypoxylon jaklitschii se caracteriza por los estromas glomerulados a efuso-pulvinados, sepia, marrón oscuro a negro, libera pigmento ocre en $\mathrm{KOH}$ y por el tamaño de sus esporas. Especies afines son $H$. lenormandii y $H$. lividipigmentum que tienen estromas glomerulados a efuso-pulvinados y colores parecidos en algunas estapas de desarrollo, pero se separan por el tamaño pequeño de las esporas de $H$. jaklitschii, con respecto a las dos especies $H$. lenormandii (11-13 × 4-6 $\mu \mathrm{m})$ y $H$. lividipigmentum (10-15 × 4.5-6 $\mu \mathrm{m})$. Por otro lado, la primera libera un pigmento magenta en $\mathrm{KOH}$, y la segunda un pigmento violeta en $\mathrm{KOH}$.

Hypoxylon lenormandii Berk. \& M.A. Curtis, J. Linn. Soc., Bot. 10 (46): 385. 1868. TIPO: CUBA. Sobre madera descortezada, s.f., C. Wright 486 (holotipo: K). Fig. 7.

= Sphaeria subaenea Berk. \& M. A. Curtis apud Berk., J. Linn. Soc., Bot. 10: 387. 360. 1869. TIPO: CUBA. Sobre madera descortezada, s.f., C. Wright 485 (holotipo: K).

= Hypoxylon oodes Berk. \& Broome, J. Linn. Soc. Bot. 14: 122. 1873. TIPO: SRI LANKA. Central Province, sobre madera descortezada, XII.1868, G. H. K. Thwaite 1085, como Sphaeria oodes (holotipo: K, isotipo: in Herbs. Cooke \& Broome).

= Rosellinia subaenea (Berk. \& M. A. Curtis) Sacc., Syll. Fung. I, p. 256. 1882.

= Hypoxylon subvinosum Speg., Anal. Soc. Cient. Argent. 18: 269. 1884. TIPO: PARAGUAY. Guarapi, sobre madera, XI.1881, B. Balansa 3423 (isotipos: BPI, K).

= Hypoxylon subaeneum (Berk. \& M. A. Curtis) Speg., Anal.

Soc. Cient. Argent. 26: 32. 1888.

= Rosellinia melaleuca Ellis \& Everh. Apud C. L. Smith, Bull. Lowa Univ. Lab. Nat. Hist. 2: 402. 1893. TIPO: NICARAGUA. Ometepe, sobre madera descortezada, I.1893, B. Shimek s.n. (isotipos: BPI, CLS).

= Kretzschmaria stilbophora Rehm, Ann. Mycol. 5: 526. 1907. TIPO: BRASIL. Rio Grande do Sul, São Leopoldo, sobre madera, s.f., J. Rick s.n. (holotipo: S, isotipo: K). 

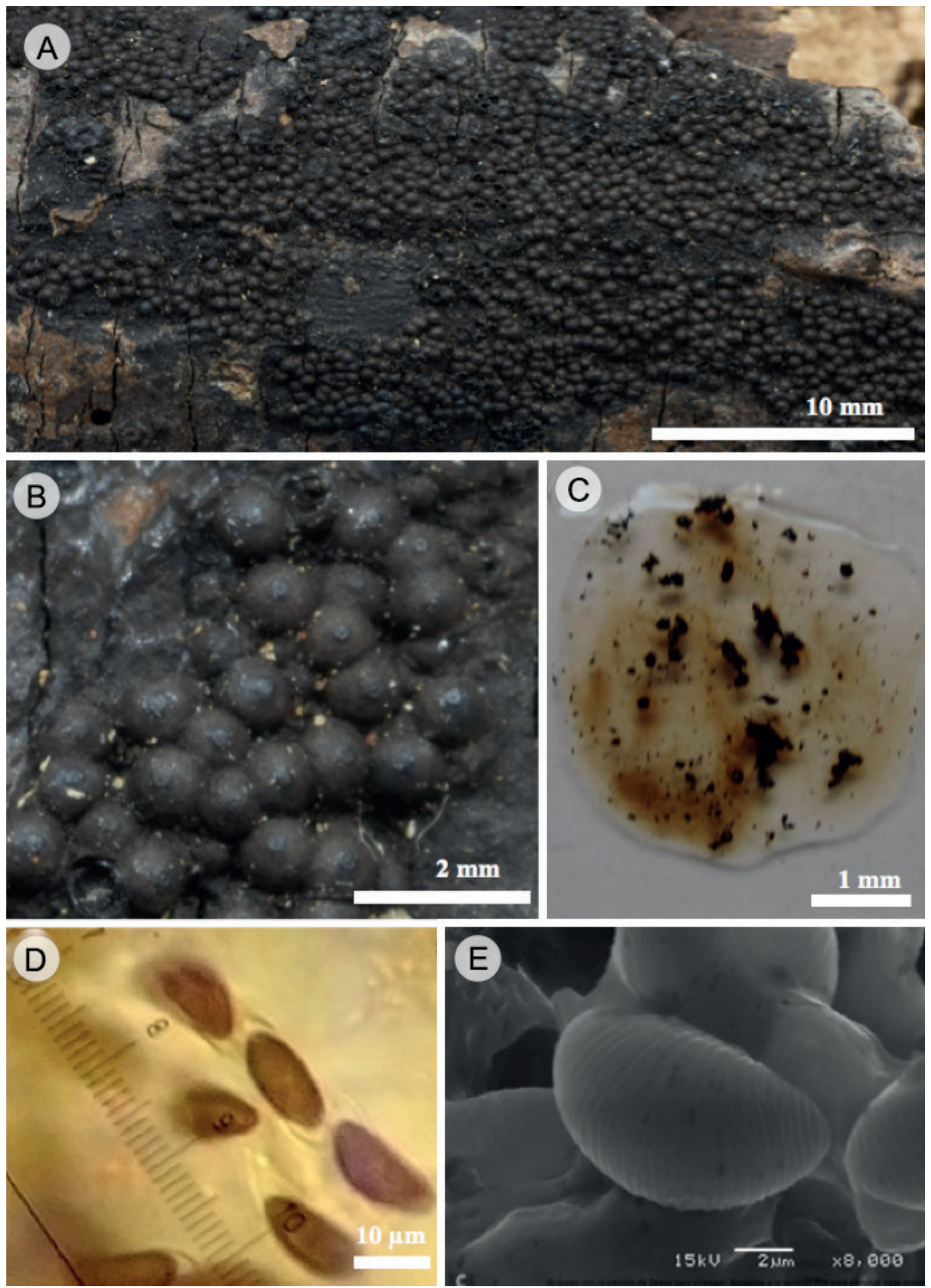

Figura 6: Hypoxylon jaklitschii Sir \& Kuhnert. A. vista general del estroma glomerulado; B. vista del estroma con los peritecios conspicuos y esféricos; C. pigmentos ocre en $\mathrm{KOH}$ al $10 \%$; D. ascosporas con perisporio dehiscente vistas en $\mathrm{MO}$; $\mathrm{E}$. ascosporas con perisporio estriado transversalmente vistas en MEB. 

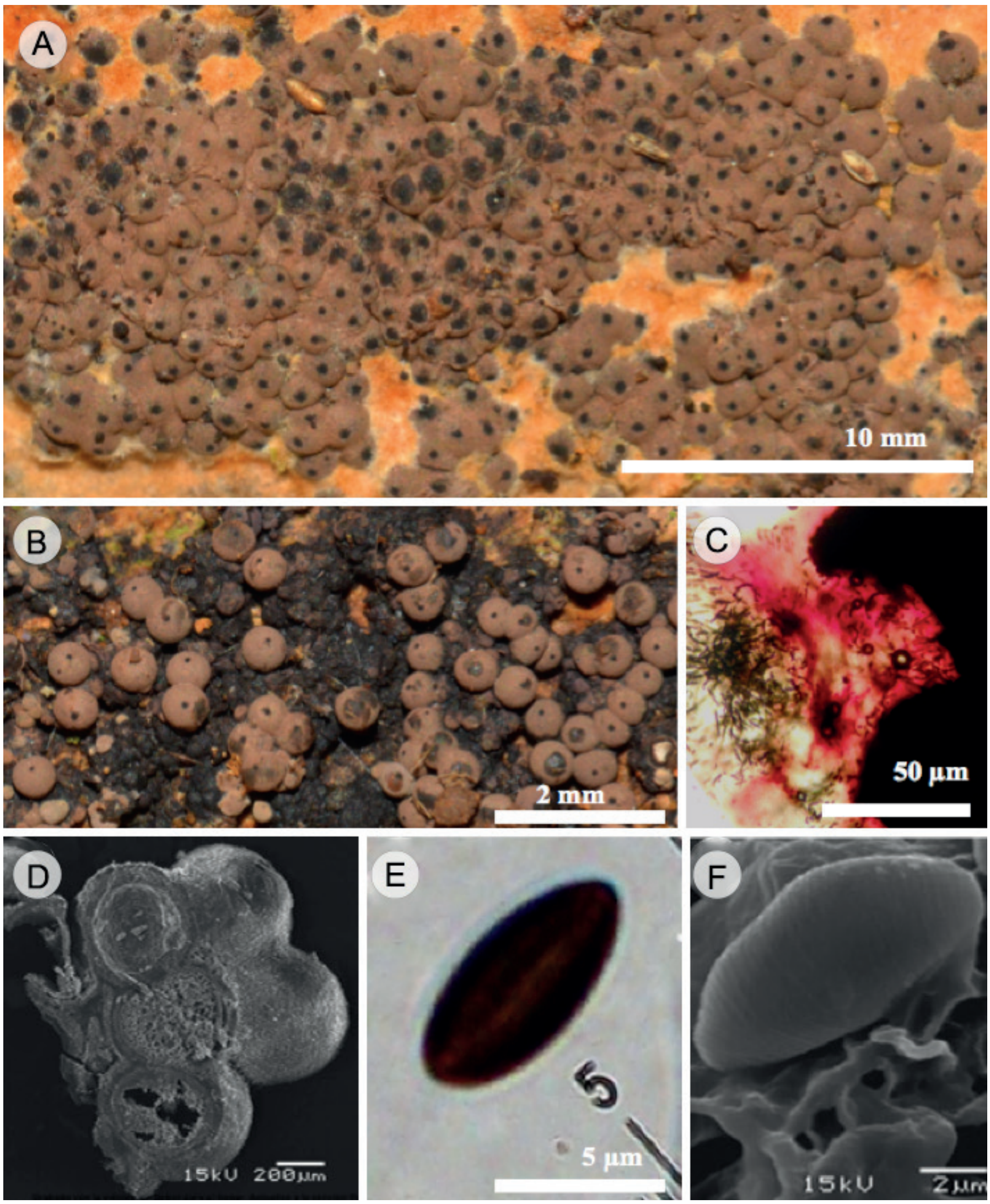

Figura 7: Hypoxylon lenormandii Berk. \& M.A. Curtis. A. vista general del estroma glomerulado; B. vista del estroma con los peritecios conspicuos y esféricos; C. pigmentos magenta en $\mathrm{KOH}$ al 10\%; D. peritecios esféricos vistos en MEB; E. ascospora con línea germinal sigmoide vistas en MO; F. ascospora con perisporio ligeramente estriado transversalmente vista en MEB. 
= Rosellinia metachroa Ferd. \& Winge, Bot. Tidsskr. 29: 16. 1908. TIPO: ESTADOS UNIDOS DE AMÉRICA. Islas Virgenes. St. John, American Hill, 17.III.1906, C. Raunkiaer s.n. (holotipo: S).

= Hypoxylon riograndense Rehm, Ann. Mycol. 7: 154. 1909. TIPO: BRASIL. São Leopoldo, sobre corteza, 1904, J. Rick s.n. (holotipo: S; isotipos: $\mathrm{BPI}, \mathrm{FH}$, sheet 7414 of Patouillard Herb.).

= Rosellinia pulchella Syd. \& P. Syd. apud Wildeman, FI. BasMoyen-Congo III: 7. 1909. TIPO: REPÚBLICA DEMOCRÁTICA DEL CONGO. Leopoldville, Kisantu, sobre madera descortezada, 31.I.1907, H. Vanderyst s.n. (holotipo: BR).

= Hypoxylon fulvoochraceum Rehm, Philipp. J. Sci. 8. 188. 1913. TIPO: FILIPINAS. Los Baños, sobre columnas de Bambusa, 14.IX.1912, C. F. Baker 50 (holotipo: S).

Estroma 10-50 × 5-30 mm, glomerulado a efuso-pulvinado, a veces roselinoide o peritecioide, aunque unidos a los peritecios por una capa de tejido estromático delgado, sepia grisáceo, marrón a marrón vináceo; endostroma con granulos marrón anaranjado a marrón oscuro; peritecios 0.4-0.5 mm de diámetro, notablemente conspicuos, a veces separados, pero sobre una superfice estromática, globosos a ovoides, ostiolos umbilicados a ligeramente papilados, desprende pigmentos magenta en $\mathrm{KOH}$ al $10 \%$, cambia a ocre después de 1 minuto de contacto; ascas 150-190 × 5-8 $\mu \mathrm{m}$, cilíndricas, uniseriadas, amiloides; ascosporas 11$13 \times 4-6 \mu \mathrm{m}$, elipsoides, inequiláteras, línea germinal sigmoide en el lado convexo, marrón oscuro, perisporio liso en $\mathrm{MO}$, inconspicuamente estriado transversalmente en MEB, dehiscente en $\mathrm{KOH}$ al $10 \%$.

Hábitat: estromas gregarios y fusionados sobre madera en descomposición de Manilkara zapota (L.) P. Royen. (conocido como chicle o chicozapote).

Distribución: se ha citado de las Antillas francesas (Fournier et al., 2015), Brasil (Cruz y Cortez, 2015), Papúa Nueva Guinea (Van der Gucht et al., 1997), Cuba, Indonesia, Nicaragua, Paraguay, Sri Lanka, Tahití y Taiwán (Ju y Rogers, 1996), Argentina, Brasil, Cuba, Estados Unidos de América, Filipinas, Indonesia, Islas Virgenes, Martinica, Paraguay, República Democrática del Congo y Tailandia (Kuhnert et al.,
2015). En México se ha citado de Campeche, Chiapas, Nayarit, Nuevo León, Oaxaca, Quintana Roo, Sinaloa, Sonora, Tamaulipas y Yucatán (Martin, 1969; Ju y Rogers, 1996; San Martín et al., 1999a; Medel, 2002; Raymundo et al., 2014, 2017). Se registra por primera vez para Jalisco.

Material examinado: MÉXICO. Jalisco, municipio La Huerta, Reserva de la Biosfera Chamela-Cuixmala, Estación de Biología Chamela, IBUNAM, camino Antiguo Sur, $19^{\circ} 29^{\prime} 45.3^{\prime \prime} \mathrm{N}, 105^{\circ} 02^{\prime} 44.84^{\prime \prime O}$, 18.IX.2011, T. Raymundo 3974 (ENCB), 3977 (ENCB), 3985 (ENCB), 4062 (ENCB); loc. cit., R. Valenzuela 14543 (ENCB), 14552 (ENCB); loc. cit., 16.IX.2012, R. Valenzuela 14471 (ENCB); loc. cit., Eje Central, $19^{\circ} 29^{\prime} 58.39^{\prime \prime} \mathrm{N}, 105^{\circ} 02^{\prime} 37.97^{\prime \prime O}$, 18.IX.2011, T. Raymundo 4974 (ENCB); loc. cit., 16.IX.2018, P. Reyes 923 (ENCB); loc. cit., Vereda Búho, $19^{\circ} 29^{\prime} 40.30^{\prime \prime} \mathrm{N}, 105^{\circ} 02^{\prime} 52.04^{\prime \prime O}$, 17.IX.2011, T. Raymundo 4013 (ENCB); loc. cit., R. Valenzuela 14498 (ENCB); loc. cit., 28.IX.2013, T. Raymundo 4764 (ENCB); loc. cit., 15.IX.2018, R. Valenzuela 18578 (ENCB); loc. cit., Vereda Chachalacas, $19^{\circ} 29^{\prime} 50.25^{\prime \prime} \mathrm{N}, 105^{\circ} 02^{\prime} 40.62^{\prime \prime O}$, 21.IX.2011, T. Raymundo 4445 (ENCB); loc. cit., Vereda Tejón, 19³0'2.94"N, 10502'41.90"O, 16.IX.2018, P. Reyes 925 (ENCB). Quintana Roo, municipio Cozumel, Reserva de la Biosfera Isla Cozumel, Zona Arqueológica San Gervasio, $20^{\circ} 29^{\prime} 58.02^{\prime \prime} \mathrm{N}, 86^{\circ} 50^{\prime} 37.88^{\prime \prime} \mathrm{O}, 20.1 .2018$, R. Valenzuela 17790 (ENCB); loc. cit., Vereda de la Fundación Parques y Museos de Cozumel, 20²8'59.19"N, 8657'21.80"O, 20.I.2018, R. Valenzuela 17957 (ENCB); 18017 (ENCB); loc. cit., Paque Ecológico Punta Sur, $28^{\circ} 16^{\prime} 48^{\prime \prime} \mathrm{N} 86^{\circ} 58^{\prime} 44.39^{\prime \prime O}$, 16.VI.2018, R. Valenzuela 18359 (ENCB). Sonora, municipio Álamos, Reserva de la Biosfera Sierra de Álamos-Río Cuchujaqui, La Sierrita, 2658'25.16"N, 10856'41.75"O, 27.X.2018, T. Raymundo 8076 (ENCB). Tamaulipas, municipio Gómez Farías, Reserva de la Biosfera El Cielo, El Nacimiento, 2241'30.18"N, 99²'50.50"O, 26.VI.2018, P. Reyes 150 (ENCB), 159 (ENCB), 162 (ENCB); loc. cit., El Azteca, $23^{\circ} 06^{\prime} 46^{\prime \prime} \mathrm{N}, 99^{\circ} 08^{\prime} 29^{\prime \prime O}, 27 . \mathrm{VI} .2018$, P. Reyes 164 (ENCB), 174 (ENCB).

Notas taxonómicas: Hypoxylon lenormandii se caracteriza por los estromas glomerulados a efuso-pulvinados, sepia grisáceo, marrón a marrón vináceo, libera pigmento magenta en $\mathrm{KOH}$ y por el tamaño de sus esporas. Kuhnert 
et al. (2015) segregaron a $H$. jaklitschii con base en datos moleculares y algunas diferencias como tamaño de esporas (más grandes en la primera) y se liberan pigmentos magenta en $\mathrm{H}$. lenormandii y ocre en $\mathrm{H}$. jaklitschii, caracteres ya discutidos en la especie anterior.

Hypoxylon lividipigmentum F. San Martín, Y.M. Ju \& J.D. Rogers, Mycol. Mem. 20: 145. 1996. TIPO: MÉXICO. Quintana Roo, municipio Othón P. Blanco, ejido La Unión, sobre madera, 8.XII.1986, San Martín 96 (holotipo: ITCV, isotipo: JDR). Fig. 8.

Estroma 10-60 × 3-10 mm, efuso-pulvinado, castaño, marrón pálido, marrón rojizo o negro, endostroma marrón con gránulos marrón rojizo; peritecios 0.2-0.4 mm diámetro, esféricos, ostiolos umbilicados, desprende pigmento violeta en $\mathrm{KOH}$ al $10 \%$, no cambia después de 1 minuto de contacto; ascas 135-160 × 6-7 $\mu \mathrm{m}$, cilíndricas, uniseriadas, octosporadas, amiloides; ascosporas 10-15 × 4.5-6 $\mu \mathrm{m}$, elipsoides, inequiláteras, línea germinal sigmoide a lo largo de la misma, marrón oscuro, perisporio liso en $\mathrm{MO}$, inconspicuamente estriado transversalmente en el centro de la espora, liso en los extremos en MEB, dehiscente en $\mathrm{KOH}$ al $10 \%$.

Hábitat: crece sobre madera en descomposición de eudicotiledónea en el bosque tropical caducifolio.

Distribución: se ha citado de Venezuela (Ju y Rogers, 1996). En México se ha citado de Oaxaca y Quintana Roo (San Martín et al., 1999a) y de Jalisco (Sánchez-Jácome y Guzmán Dávalos, 2005). Se registra por primera vez para Sonora y Tamaulipas.

Material examinado: MÉXICO. Jalisco, municipio La Huerta, Reserva de la Biosfera Chamela-Cuixmala, Estación de Biología Chamela, IBUNAM, Eje Central, 19²9'58.39"N, $105^{\circ} 02$ '37.97"O, 16.IX.2018, P. Reyes 923 (ENCB), 925 (ENCB); loc. cit., Vereda Búho, $19^{\circ} 29^{\prime} 40.30^{\prime \prime} \mathrm{N}, 105^{\circ} 02^{\prime} 52.04^{\prime \prime O}$, 15.IX.2018, P. Reyes 917 (ENCB). Sonora, municipio Álamos, Reserva de la Biosfera Sierra de Álamos-Río Cuchujaqui, La Sierrita, $26^{\circ} 58^{\prime} 25.16^{\prime \prime} \mathrm{N}, 108^{\circ} 56^{\prime} 41.75^{\prime \prime O}$, 8.X.2014, R. Valenzuela 15870 (ENCB); loc. cit., 9.X.2014, T. Raymundo 5489 (ENCB); loc. cit., R. Valenzuela 16086 (ENCB); loc. cit.,
Promontorios, $27^{\circ} 27^{\prime} 54.1^{\prime \prime} \mathrm{N}, 108^{\circ} 45^{\prime} 22.76^{\prime \prime O}, 7 . X .2014, T$. Raymundo 5401 (ENCB). Tamaulipas, municipio Gómez Farías, Reserva de la Biosfera El Cielo, La Florida 22 $59^{\prime} 22^{\prime \prime} \mathrm{N}$, 9908'35"O, 26.VI.2018, P. Reyes 171 (ENCB).

Notas taxonómicas: Hypoxylon lividipigmentum es una especie afín a H. lividicolor Y.M. Ju \& J.D. Rogers en la forma y color del estroma, así como en el color violeta de los pigmentos. Se separan porque $H$. lividicolor tiene ascosporas más pequeñas de 11-12.5 × 4-4.5 $\mu$ m.

Hypoxylon perforatum (Schwein.) Fr., Summa veg. Scand., Sectio Post.: 384. 1849. Fig. 9.

ISphaeria perforata Schwein., Schr. naturf. Ges. Leipzig 1: 31. 1822. TIPO: ESTADOS UNIDOS DE AMÉRICA. Pennsylvania, Northampton County, Bethlehem, sobre corteza, s.f., L.D. Schweinitz 1194 (lectotipo: PH, designado por Ju y Rogers, 1996). Carolina del Norte, Salem, sobre corteza, s.f., Collins 28 (isolectotipos: PH, BPI).

= Sphaeria durissima Schwein., Schr. naturf. Ges. Leipzig 1: 32. 1822. TIPO: ESTADOS UNIDOS DE AMÉRICA. Pennsylvania, Northampton County, Bethlehem, sobre corteza, s.f., L.D. Schweinitz 1174 (holotipo: PH). Carolina del Norte, Salem, sobre corteza, s.f., Collins 20 (isotipos: PH, BPI).

= Sphaeria catalpa Schwein., Trans. Am. phil. Soc., New Series 4(2): 193. 1832. TIPO. ESTADOS UNIDOS DE AMÉRICA. Pennsylvania, Northampton County, Bethlehem, sobre corteza podrida de Catalpa, s.f., L. D. Schweinitz 1214 (holotipo: $\mathrm{PH}$, ) s.f., Collins 42 (isotipos:PH, BPI).

= Sphaeria decorticata Schwein., Trans. Am. phil. Soc., New Series 4(2): 191. 1832. TIPO. ESTADOS UNIDOS DE AMÉRICA. Nueva Inglaterra, sobre madera descortezada de Fagus, s.f., J. Torrey s.n. (holotipo: PH). Pennsylvania, Bethlehem, sobre madera descortezada de Fagus, s.f., L. D. Schweinitz 1179 (isotipo: BPI).

= Sphaeria leucostigma Lév., Annls Sci. Nat., Bot., sér. 3 9: 142. 1848. TIPO: FRANCIA. Vincennes, cerca de París, en corteza de Quercus, s.f., sin colector s.n. (holotipo: PC).

= Hypoxylon decorticatum M.A. Curtis, Geol. Nat. Hist. Surv. N. Carol., Pt 3: 140. 1867.

= Hypoxylon catalpa (Schwein.) Sacc., Syll. fung. (Abellini) 1: 392. 1882. 

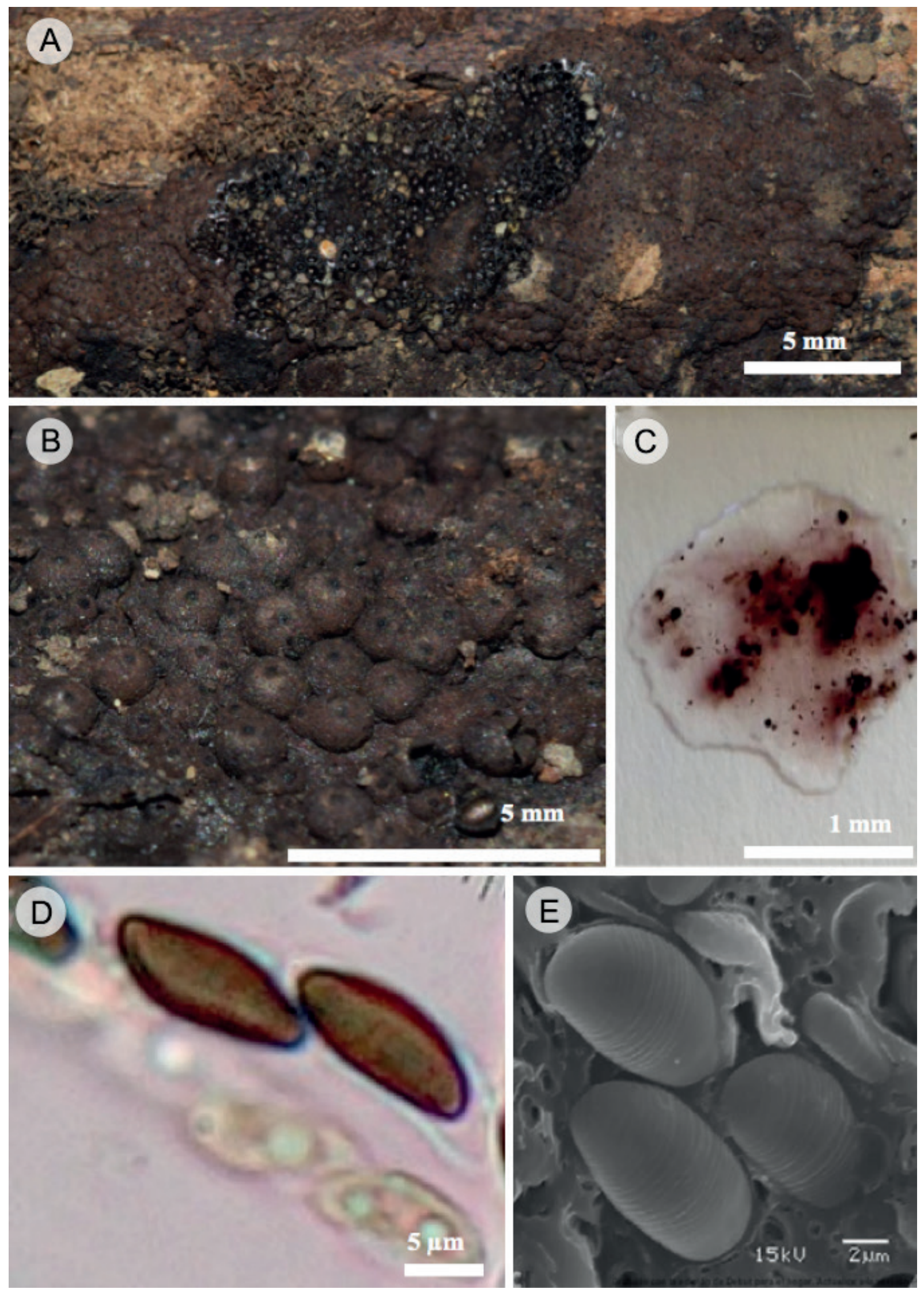

Figura 8: Hypoxylon lividipigmentum F. San Martín, Y.M. Ju \& J.D. Rogers. A. vista general del estroma efuso-pulvinado; B. vista del estroma con los peritecios conspicuos y esféricos; C. pigmentos violetas en $\mathrm{KOH}$ al 10\%; D. ascosporas con perisporio dehiscente y con línea germinal sigmoide vistas en $\mathrm{MO}$; E. ascosporas con perisporio estriado transversalmente vistas en MEB. 

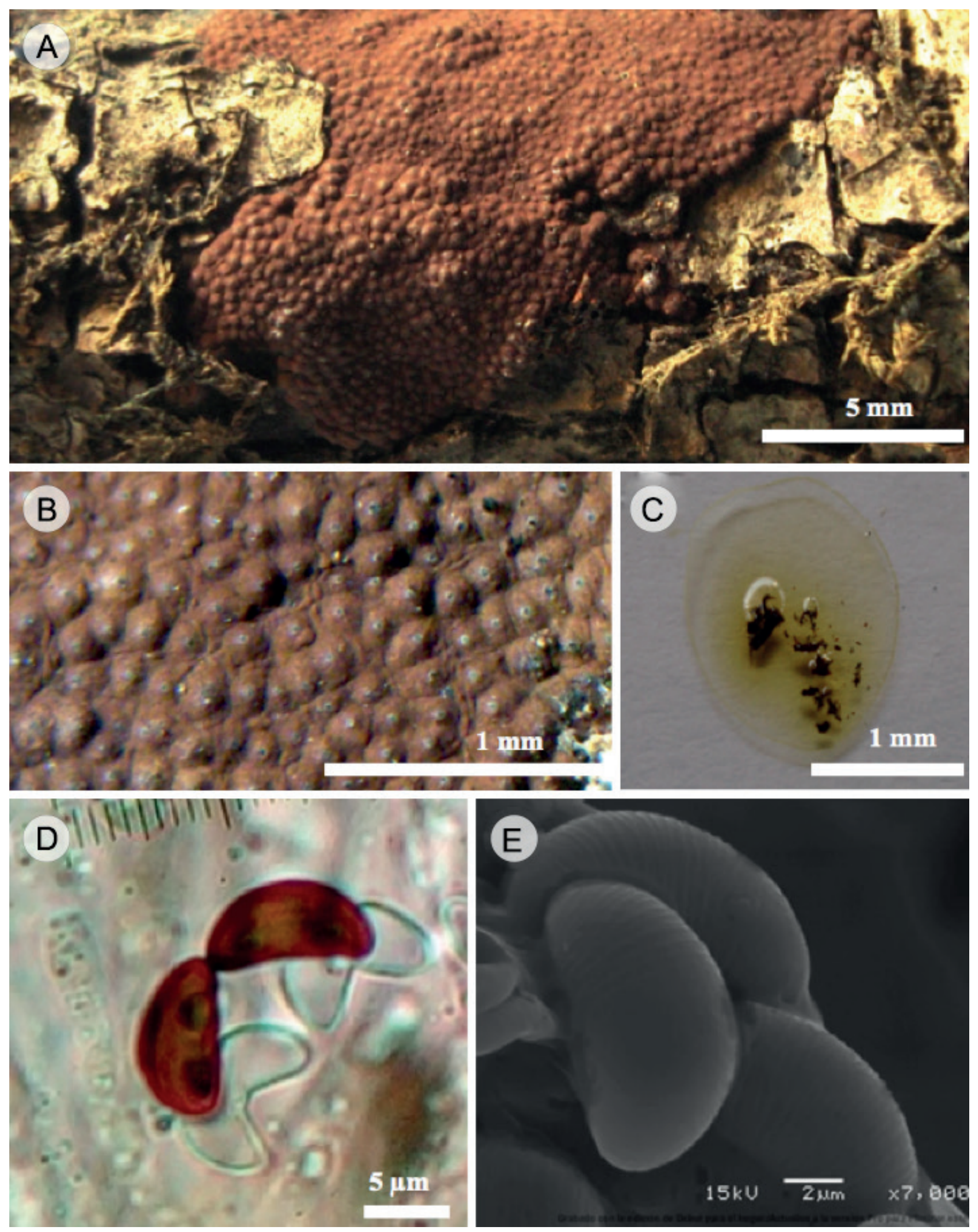

Figura 9: H. perforatum (Schwein.) Fr. A. vista general de estroma efuso-pulvinado; B. estroma mostrando ostiolos umbilicados rodeados por disco color blanco; C. pigmentos ámbar en $\mathrm{KOH}$ al 10\%; D. ascosporas con perisporio dehiscente vistas en $\mathrm{MO}$; E. ascosporas con perisporio estriado transversalmente vistas en MEB. 
= Hypoxylon durissimum (Schwein.) Sacc., Syll. fung. (Abellini) 1: 378. 1882

= Hypoxylon leucostigma (Lev.) Sacc., Syll. fung. (Abellini) 1: 367.1882.

= Hypoxylon luridum Nitschke, Pyrenomyc. Germ. 1: 31. 1867. TIPO. ALEMANIA. Westfalen, Wilkinghege in der Umgeburg von Münster, en corteza de ramas de Corylus, V.1864, T. Nitschke s.n. (holotipo: B).

= Hypoxylon plumbeum Speg., Anal. Soc. Cient. Argent. 18(6): 270. 1884. TIPO: PARAGUAY. Guarapi, en corteza de madera dura, 2.VIII.1881, B. Balansa 2760 (isotipo: BPI).

= Hypoxylon rubiginosum var. microcarpum Speg., Anales Mus. Nac. B. Aires, Ser. 3 17(10): 120. 1908. TIPO: ARGENTINA. San Pedro, Misiones, en corteza de llex paraguayensis, II.1907, C. L. Spegazzini s.n. (holotipo: LPS2017).

इ Hypoxylon rubiginosum var. perforatum (Schwein.) L.E. Petrini, Mycol. Helv. 1(7): 531. 1986.

Estroma 10-40 × 6-20 mm, efuso-pulvinado, marrón rojizo a marrón vináceo, endostroma con granulos amarillos a amarillo ocráceos; peritecios 0.1-0.3 mm de diámetro, esféricos, ostiolos umbilicados rodeados por un disco color blanco, desprende pigmentos ámbar a amarillo verdosos en $\mathrm{KOH}$ al $10 \%$, no cambia después de 1 minuto de contacto; ascas 80-130 × 5-6 $\mu \mathrm{m}$, cilíndricas, uniseriadas, octosporadas, amiloides; ascosporas 9-12 × 4-6 $\mu \mathrm{m}$, marrón oscuro, elipsoides, inequiláteras, extremos redondeados, línea germinal recta a lo largo de la misma, perisporio liso en $\mathrm{MO}$, inconspicuamente estriado transversalmente en MEB, dehiscente en $\mathrm{KOH}$ al $10 \%$.

Hábitat: estroma solitario sobre madera en descomposición de eudicotiledónea en bosque tropical caducifolio.

Distribución: se ha citado de Papúa Nueva Guinea (Van der Gucht et al., 1997), Hawái (Rogers y Ju, 2012), Alemania, Brasil, Francia, India, Madagascar, Nueva Zelanda, Paraguay, Suiza y Taiwán (Ju y Rogers, 1996). En México se ha citado de Nuevo León (San Martín et al., 1999a). Se registra por primera vez para Jalisco y Quintana Roo.

Material examinado: MÉXICO. Jalisco, municipio La Huerta, Reserva de la Biosfera Chamela-Cuixmala, Esta- ción de Biología Chamela, IBUNAM, camino Antiguo Sur, $19^{\circ} 29^{\prime} 45.3^{\prime \prime} \mathrm{N}, 105^{\circ} 02^{\prime} 44.84^{\prime \prime O}, 22 . I X .2012$, T. Raymundo 4439 (ENCB). Quintana Roo, municipio Cozumel, Reserva de la Biosfera Isla Cozumel, Zona Arqueológica San Gervasio, 20²9'58.02"N, 8650'37.88"O, 19.I.2018, P. Reyes 139 (ENCB); loc. cit., Vereda de la Fundación Parques y Museos de Cozumel, 20²8'59.19' N, 8657'21.80'O, 20.I.2018, P. Reyes 141 (ENCB), 146 (ENCB); loc. cit., R. Valenzuela 17942 (ENCB).

Notas taxonómicas: Hypoxylon perforatum se caracteriza por sus estromas efuso-pulvinados, marrón rojizos a marrón vináceo, liberan un pigmento ambar a amarillo verdoso, ostiolos rodeados por un disco blanco y el tamaño de las esporas. Una especie afín es Hypoxylon anthochroum por su similitud en forma y color de los estromas y tamaño de las esporas, pero se separan por la ausencia de discos blanquecino que rodean los ostiolos y por el color de los pigmentos extraíbles los cuales son oliváceos en $\mathrm{H}$. anthochroum y amarillos verdosos a ámbar en H. perforatum.

Hypoxylon rickii Y.M. Ju \& J.D. Rogers, Mycol. Mem. 20: 174. 1996. TIPO: BRASIL. Sobre madera descortezada, s.f., J. Rick 303 (holotipo: NY). Fig. 10.

Estroma 20-80 × 10-40 mm, efuso a efuso-pulvinado, anaranjado a anaranjado rojizo, endostroma con gránulos anaranjados; peritecios 0.6-0.8 $\times$ 0.3-0.4 mm, tubulares, ostiolos umbilicados, desprende pigmentos anaranjados a rojo escarlata en $\mathrm{KOH}$ al 10\%, no cambia después de 1 minuto de contacto; ascas 100-125 × 4-6 $\mu \mathrm{m}$, cilíndricas, uniseriadas, octosporadas, amiloides; ascosporas 6.8-7.4 $\times$ 3.2-3.7 $\mu \mathrm{m}$, elipsoides, inequiláteras, extremos redondeados, línea germinal recta a lo largo de la misma, marrón oscuro, perisporio liso en $\mathrm{MO}$, inconspicuamente estriado transversalmente en MEB, dehiscente en $\mathrm{KOH}$ al $10 \%$.

Hábitat: estromas gregarios a fusionados sobre madera en descomposición de eudicotiledónea en bosque tropical caducifolio.

Distribución: se ha citado de las Antillas francesas (Fournier et al., 2015), Brasil (Cruz y Cortez, 2015) y Guyana 

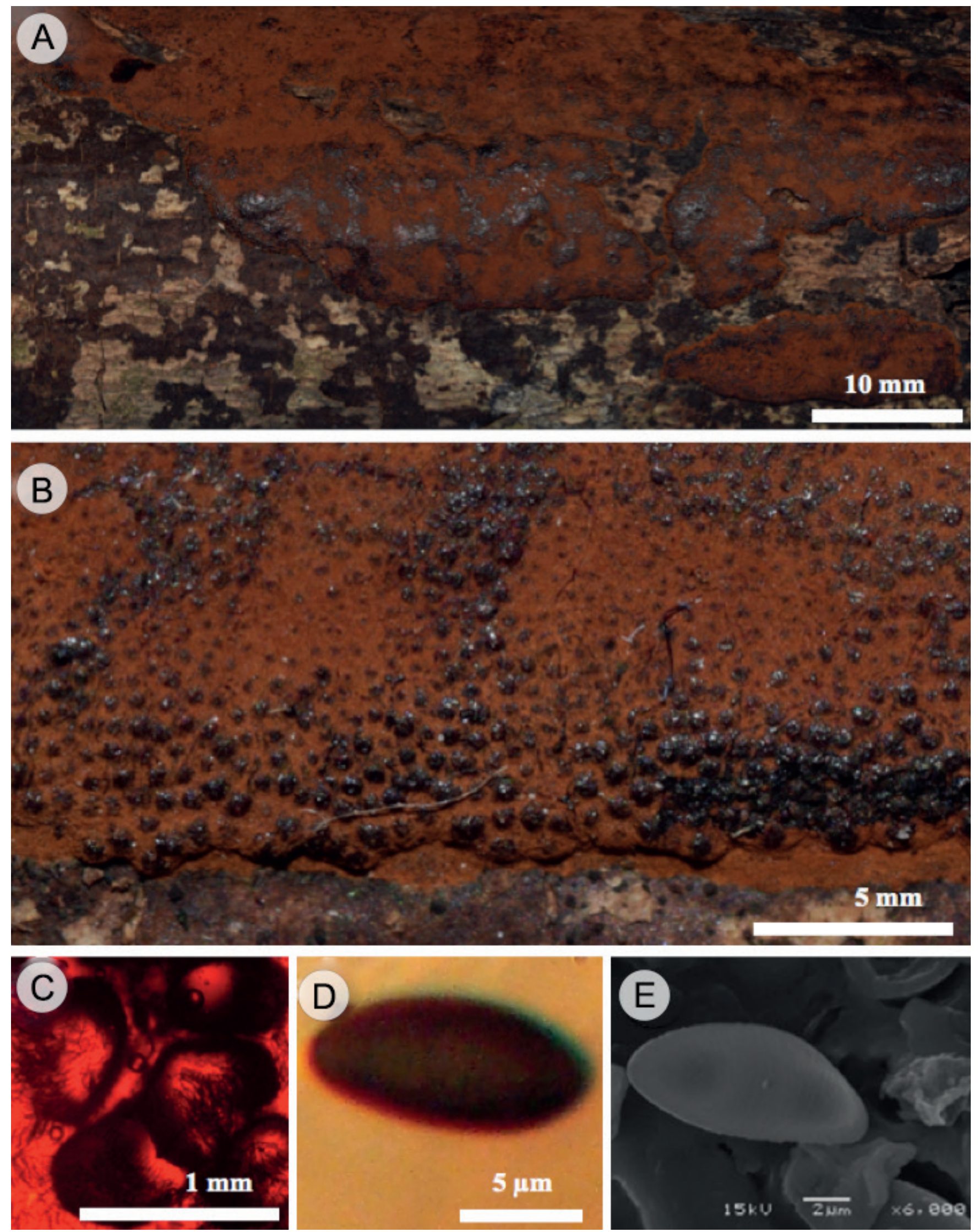

Figura 10: Hypoxylon rickii Y.M. Ju \& J.D. Rogers. A y B. vista general del estroma efuso-pulvinado; C. corte transversal de peritecios mostrando pigmentos rojo escarlata en $\mathrm{KOH} 10 \%$; D. ascospora con línea germinal recta vista en $\mathrm{MO}$; E. ascospora con perisporio ligeramente estriado vista en MEB. 
francesa (Ju y Rogers, 1996). En México se ha citado de Oaxaca (San Martín et al., 1999a). Se registra por primera vez de Quintana Roo, Sonora y Tamaulipas.

Material examinado: MÉXICO. Quintana Roo, municipio Cozumel, Reserva de la Biosfera Isla Cozumel, Zona Arqueológica San Gervasio, 20²9'58.02"N, 8650'37.88"O, 21.I.2018, P. Reyes 149 (ENCB); loc. cit., 19.I.2018, R. Valenzuela 17970 (ENCB); loc. cit., Vereda de la Fundación Parques y Museos de Cozumel, 20²8'59.19"N, 8657'21.80"O, 20.I.2018, R. Valenzuela 18012 (ENCB). Sonora, municipio Álamos, Reserva de la Biosfera Sierra de Álamos-Río Cuchujaqui, El Cajón, $27^{\circ} 01^{\prime} 41.58^{\prime \prime} \mathrm{N}, 108^{\circ} 45^{\prime} 22.76^{\prime \prime} \mathrm{O}$, 14.X.2013, R. Valenzuela 17122 (ENCB); loc. cit., La Sierrita, $26^{\circ} 58^{\prime} 25.16^{\prime \prime} \mathrm{N}, 108^{\circ} 56^{\prime} 41.75^{\prime \prime} \mathrm{O}, 8 . X .2014, R$. Valenzuela 15863 (ENCB). Tamaulipas, municipio de Gómez Farías, Reserva de la Biosfera El Cielo, La Florida, 2259'22"N, 9908'35"O, 5.X.2017, P. Reyes 100 (ENCB).

Notas taxonómicas: Hypoxylon rickii es una especie afín a $H$. cinnabarinum, $H$. fendleri y $H$. haematostroma por presentar estromas y pigmentos extraíbles similares. Se separa de esas especies porque tienen las ascosporas más grandes, la primera con esporas 9.5-14 × 5-7.5 $\mu \mathrm{m}$, la segunda con esporas (8-) 9-12 $\times$ 4-5.5 $\mu \mathrm{m}$, la tercera con esporas 13.5-18 (-19) $\times$ 7-8(-8.5) $\mu \mathrm{m}$. Las esporas de H. rickii miden 6.8-7.4 × 3.2-3.7 $\mu \mathrm{m}$, y son las más pequeñas del complejo.

Hypoxylon subgilvum Berk. \& Broome, J. Linn. Soc., Bot. 14(74): 120. 1873. TIPO: SRI LANKA. Central Province, sobre corteza, XII.1868, G. H. K. Thwaite 1087 (holotipo: K). Fig. 11.

= Hypoxylon caaguazu Speg., Anal. Soc. Cient. Argent. 18(6): 275. 1884. TIPO: PARAGUAY. Sobre madera muerta, I.1882, B. Balansa 3359-60 (isotipo: BPI).

= Hypoxylon hypomiltum var. microsporum Y. Abe, Trans. Mycol. Soc. Japan 27: 55. 1986. TIPO: JAPÓN. Kitakata, Higashiusuki-gun, Miyazaki Pref., en corteza de Quercus acutissima, 29.IX.1976, K. Aoshima y M. Tsunoda TFM-F-12665 (holotipo: TFM).

= Hypoxylon subgilvum var. microsporum (Y. Abe) Y.M. Ju \& J.D. Rogers, Mycol. Mem. 20: 188. 1996.
Estroma 10-40 × 4-8 mm, efuso-pulvinado, marrón rojizo a marrón vinaceo, endostroma con gránulos anaranjados a anaranjado rojizos; peritecios 0.2-0.6 $\mathrm{mm}$ de diámetro, conspicuos, esféricos a ovoides, ostiolos umbilicados, desprende pigmentos anaranjado amarillentos a anaranjado rojizos en $\mathrm{KOH}$ al $10 \%$, no cambia después de 1 minuto de contacto; ascas 90-150 × 4-7 $\mu \mathrm{m}$, cilíndricas, uniseriadas, octosporadas, amiloides; ascosporas 8-11 $\times$ 4-5 $\mu \mathrm{m}$, elipsoides, inequiláteras, extremos redondeados, línea germinal recta a lo largo de la misma, marrón oscuro, perisporio liso en $\mathrm{MO}$, conspicuamente estriado transversalmente en MEB, dehiscente en $\mathrm{KOH}$ al $10 \%$.

Hábitat: estromas solitarios a gregarios a fusionados, sobre madera de eudicotiledónea en bosque tropical caducifolio.

Distribución: se ha citado de las Antillas francesas (Fournier et al., 2015), Brasil (Cruz y Cortez, 2015), Hawái (Rogers y Ju, 2012); Tailandia (Vasilyeva et al., 2012); Papúa Nueva Guinea (Van der Gucht et al., 1997); Congo, India, Indonesia, Paraguay, Sudáfrica, Taiwán y Venezuela (Ju y Rogers, 1996). En México ha sido citado de Tamaulipas y Campeche (San Martín et al., 1999a). Se registra por primera vez para Jalisco y Quintana Roo.

Material examinado: MÉXICO. Jalisco, municipio La Huerta, Reserva de la Biosfera Chamela-Cuixmala, Estación de Biología Chamela, IBUNAM, Vereda Búho, $19^{\circ} 29^{\prime} 40.30 " \mathrm{~N}, 105^{\circ} 02^{\prime} 52.04^{\prime \prime O}$, 17.IX.2011, P. Garma 645 (ENCB); loc. cit., 16.IX.2018, P. Reyes 914 (ENCB). Quintana Roo, municipio Cozumel, Reserva de la Biosfera Isla Cozumel, Zona Arqueológica San Gervasio, 20²9'58.02"N, 8650'37.88"O, 19.I.2018, R. Valenzuela 18011 (ENCB); loc. cit., T. Raymundo 7334 (ENCB); loc. cit., Vereda de la Fundación Parques y Museos de Cozumel, 20²8'59.19"N, 8657'21.80"O, 20.I.2018, T. Raymundo 7307 (ENCB). Tamaulipas, municipio Gómez Farías, Reserva de la Biosfera EI Cielo, camino San José - La Gloria, La Alamillosa, $23^{\circ} 03^{\prime} 20^{\prime \prime} \mathrm{N}$, 99¹2'46"O, 6.X.2017, P. Reyes 101 (ENCB); loc. cit., El Nacimiento, 22 $41^{\prime} 30.18^{\prime \prime} \mathrm{N}, 9^{\circ} 2^{\prime} 50.50 " \mathrm{O}, 25 . \mathrm{VI} .2018$, P. Reyes

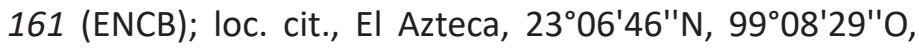
27.VI.2018, P. Reyes 166 (ENCB). 

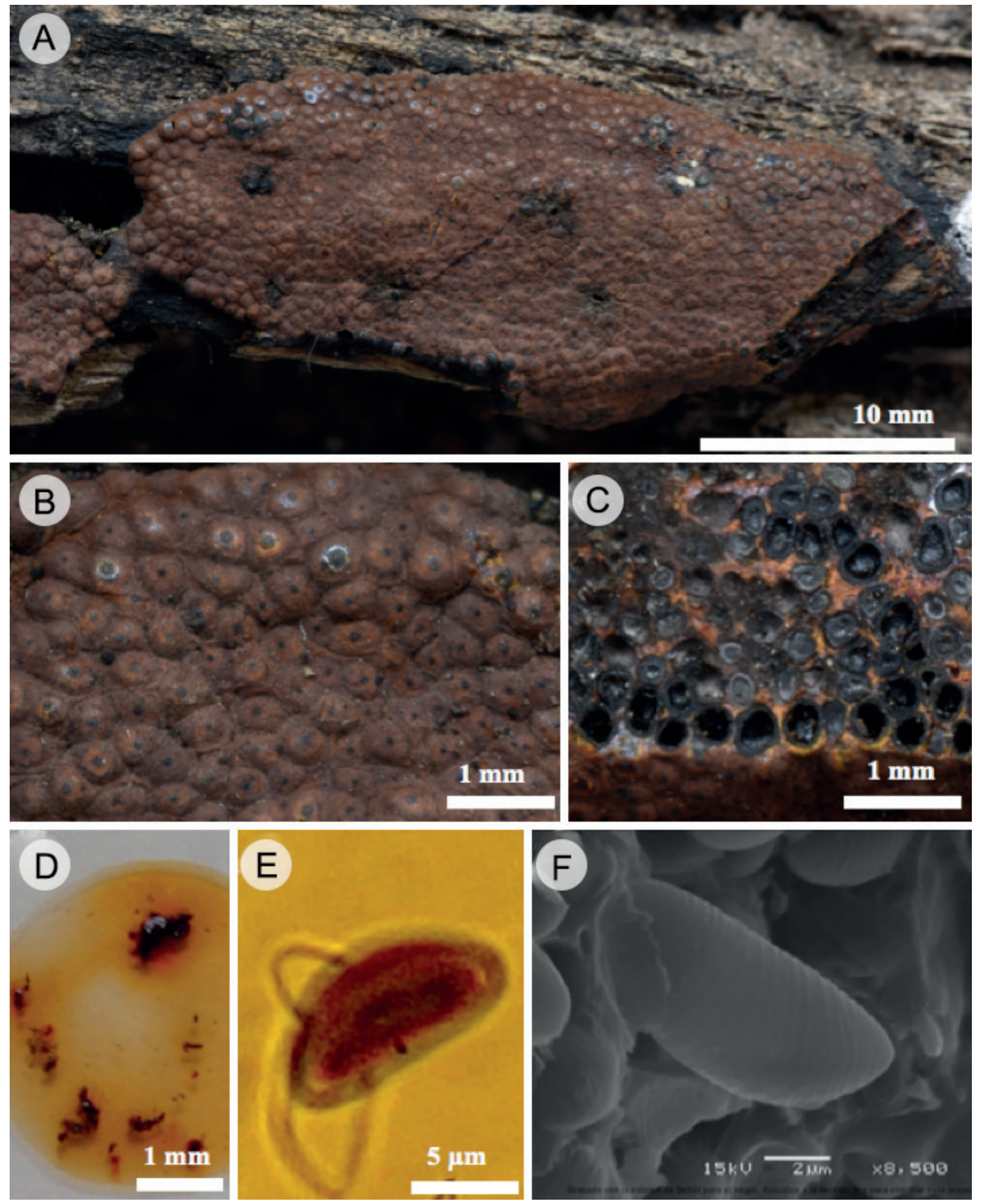

Figura 11: Hypoxylon subgilvum Berk. \& Broome. A y B. vista general del estroma efuso-pulvinado; C. estroma en corte mostrando peritecios esféricos y gránulos endostromatales anaranjados; D. pigmentos anaranjados en $\mathrm{KOH}$ al 10\%; E. ascospora con perisporio dehiscente vista en $\mathrm{MO}$; F. ascospora con perisporio estriado transversalmente vista en MEB. 
Notas taxonómicas: Hypoxylon subgilvum presenta un estroma marrón a marrón vináceo con gránulos anaranjados a anaranjado rojizos en el endostroma y los pigmentos extraíbles anaranjados a rojizos que la hacen afín a $H$. crocopeplum Berk. \& M.A. Curtis, H. fendleri e H. samuelsii Y.M. Ju \& J.D. Rogers. Sin embargo, es fácil diferenciarlas por los tipos de esporas que presentan las cuatro especies; $H$. fendleri tiene las esporas de 9-11 × 4-5 $\mu \mathrm{m}$, muy similares a H. subgilvum, pero la hendidura longitudinal es ligeramente sigmoide, separándose de las otras tres especies por tener la hendidura longitudinal recta. Por otro lado, Hypoxylon crocopeplum tiene las esporas son mas grandes $(9.5-15 \times 4-7 \mu \mathrm{m})$ que la especie aquí estudiada e $H$. samuelsii sus esporas son mas angostas que las otras especies (7.3-9.8 × 2.8-3.4 $\mu \mathrm{m})$.

\section{Discusión}

Las especies de Hypoxylon se pueden diferenciar fácilmente por la forma y color del estroma, así como por los pigmentos que liberan en $\mathrm{KOH}$. Con base en estas características se dividen en diferentes grupos morfológicos; primero, tenemos a los de estroma anaranjado y pigmentos rojizo anaranjado: $H$. haematostroma, $H$. cinnabarinum, $H$. rickii con un toque anaranjado escarlata, aunque el tamaño de las ascosporas varía entre ellas: $13-17(-19) \times 7-8 \mu \mathrm{m}$ en $\mathrm{H}$. haematostroma, 10-12 × 5-6 $\mu \mathrm{m}$ en $H$. cinnabarinum y 6.8-7.4 × 3.2-3.7 $\mu \mathrm{m}$ en $H$. rickii. Después tenemos a las de estroma de tonos marrón, Hypoxylon perforatum e $H$. anthochroum, dos especies bastante parecidas entre sí, al presentar el disco blanquecino que rodea a los ostiolos en $H$. perforatum, caracter que está ausente en $H$. anthochroum. Además, el color de los pigmentos en la primera es de un tono ámbar y en la segunda son oliváceos. Hypoxylon subgilvum y $\mathrm{H}$. erythrostroma se caracterizan por los pigmentos estromáticos anaranjado amarillentos a anaranjado rojizos y los gránulos endostromatales anaranjados a anaranjado rojizos, pero el estroma efuso-pulvinado marrón rojizo a marrón vinaceo con peritecios evidentemente conspicuos en el primero, se distinguen del estroma marrón a sepia grisáceo con peritecios casi inconspicuos del segundo. Finalmente, en cuanto a las glomeruladas como H. lenormandii e H. jaklitschii: el color del estroma en la primera es marrón claro con pigmentos magenta y en la segunda es de coloración marrón oscuro con pigmentos ocres.

En el Cuadro 2 podemos observar que las especies que se encontraron en todas las ANPs son Hypoxylon cinnabarinum, $H$. haematostroma e $H$. lenormandii. Hypoxylon

Cuadro 2: Especies del género Hypoxylon Bull. por Área Natural Protegida con bosque tropical caducifolio en México.

\begin{tabular}{|c|c|c|c|c|}
\hline Especie/ Reserva de la Biosfera & $\begin{array}{l}\text { RB Chamela- } \\
\text { Cuixmala, Jalisco }\end{array}$ & $\begin{array}{l}\text { RB Isla Cozumel, } \\
\text { Quintana Roo }\end{array}$ & $\begin{array}{c}\text { RB Sierra de Álamos- } \\
\text { Río Cuchujaqui, } \\
\text { Sonora }\end{array}$ & $\begin{array}{l}\text { RB El Cielo, } \\
\text { Tamaulipas }\end{array}$ \\
\hline H. anthochroum Berk. \& Broome & $x$ & & $x$ & \\
\hline H. cinnabarinum (Henn.) Henn. & $x$ & $x$ & $x$ & $x$ \\
\hline H. erythrostroma J. H. Mill. & & $x$ & $x$ & $x$ \\
\hline H. haematostroma Mont. & $x$ & $x$ & $x$ & $x$ \\
\hline H. investiens (Schwein.) M.A. Curtis & & $x$ & $x$ & $x$ \\
\hline H. jaklitschii Sir \& Kuhnert & $x$ & $x$ & & $x$ \\
\hline H. lenormandii Berk. \& M.A. Curtis & $x$ & $x$ & $x$ & $x$ \\
\hline $\begin{array}{l}\text { H. lividipigmentum F. San Martín, Y.M. Ju \& J.D. } \\
\text { Rogers }\end{array}$ & $x$ & & $x$ & $x$ \\
\hline H. perforatum (Schwein.) Fr. & $x$ & $x$ & & \\
\hline H. rickii Y.M. Ju \& J.D. Rogers & & $x$ & $x$ & $x$ \\
\hline H. subgilvum Berk. \& Broome & $x$ & $x$ & & $x$ \\
\hline
\end{tabular}


erythrostroma, $H$. jaklitschii, $H$. investiens, $H$. lividipigmentum, $H$. rickii e $H$. subgilvum se encontraron en tres, mientras que se registraron a $H$. anthochroum y $H$. perforatum en dos. En cuanto al número de especies por ANPs, no se presentaron diferencias en cuanto a su diversidad, porque la Reserva de la Biosfera El Cielo y la Reserva de la Biosfera Isla de Cozumel tuvieron nueve especies, y la Reserva de la Biosfera Álamos-Río Cuchujaqui y la Reserva de la Biosfera Chamela-Río Cuixmala presentaron ocho.

\section{Conclusiones}

En total se determinaron 11 taxones de Hypoxylon que crecen en el bosque tropical caducifolio de México en cuatro Áreas Naturales Protegidas. Una es nuevo registro para México $(H$. jaklitschii recolectada en tres de las ANP's: Jalisco, Quintana Roo y Tamaulipas), aumentándose a 37 las especies para dicho género. De las 11 especies determinadas, Hypoxylon jaklitschii, H. perforatum, H. rickii e Hypoxylon subgilvum no habían sido citadas del bosque tropical caducifolio, por lo que se aumenta a 24 taxones de Hypoxylon para este tipo de vegetación. Las especies que se encontraron en todas las ANPs son $H$. cinnabarinum, H. haematostroma e $\mathrm{H}$. lenormandii. Esta última fue la de mayor frecuencia al presentar 25 especímenes, seguida de $H$. haematostroma con 20. Nueve de las especies de Hypoxylon estudiadas presentan distribución pantropical, mientras que $H$. lividipigmentum e $\mathrm{H}$. rickii son especies exclusivamente neotropicales. Finalmente, podemos concluir que las ANPs, con sus bosques de tipo tropical caducifolios, son muy diversas en especies de Hypoxylon y se requiere ampliar el número de exploraciones a éstas y otras ANPs o realizar estudios en otras zonas con este tipo de vegetación, el cual se extiende por toda la costa del Pacífico y en la planicie costera del Golfo de México.

\section{Contribución de autores}

PERR, RV y TR concibieron y diseñaron el estudio. Todos los autores realizaron las recolecciones y determinaciones de las especies. Las fotos de las figuras fueron tomadas por RV y TR y las de MEB por PERR. PERR redactó el manuscrito con la ayuda de TR. Todos los autores contribuyeron a la discusión, revisión y aprobación del manuscrito final.

\section{Financiamiento}

Este estudio fue apoyado por el Instituto Politécnico Nacional a través de la Secretaría de Investigación y Posgrado en los proyectos (SIP): 20180243, 20180244, 20195221, 20195222, 20200248, 20200956. Los autores agradecen al Consejo Nacional de Ciencia y Tecnologia por el financiamento al proyecto 252934 de la convocatoria Ciencia Básica Secretaría de Educacion Pública - Consejo Nacional de Ciencia y Tecnologia 2015. PERR es becaria del Consejo Nacional de Ciencia y Tecnología para realizar sus estudios de Maestría en Biociencias en la Escuela Nacional de Ciencias Biológicas del Instituto Politécnico Nacional y contó con la beca de Estímulo Institucional de Formación de Investigadores (BEIFI). RV y TR recibieron becas para realizar sus investigaciones de la Comisión de Operación y Fomento de las Actividades Académicas del IPN, la Beca Estimulo al Desempeño a la Investigación de la Secretaria de Posgrado e Investigación del IPN, y del Sistema Nacional de Investigadores.

\section{Agradecimientos}

Los autores agradecen a las autoridades de la Escuela Nacional de Ciencias Biológicas (ENCB) del Instituto Politécnico Nacional las facilidades otorgadas para realizar el presente estudio y a los encargados de la ANPs por las facilidades otorgadas.

\section{Literatura citada}

Angulo, A., J. Rueda-Almonacid, J. Rodríguez-Mahecha y E. La Marca. 2006. Técnicas de inventario y monitoreo para los anfibios de la región tropical andina. Conservación Internacional. Serie Manuales de Campo n.ㅇ 2. Panamericana Formas e Impresos S.A. Bogotá, D.C., Colombia. Pp. 135-137.

Arriaga, L., J. Espinoza, C. Aguilar, E. Martínez, L. Gómez y E. Loa. 2000. Regiones terrestres prioritarias de México. Comisión Nacional para el Conocimiento y Uso de la Biodiversidad. México, D.F., México. 301 pp.

Ávalos-Lázaro, A., J. Rosique, S. Cappello y J. L. Villarruel. 2018. Ascomicetes (Fungi: Ascomycota) del Parque Estatal Agua Blanca, Macuspana, Tabasco, México. Acta Botanica Mexicana 122: 141-154. DOI: https://doi.org/10.21829/ abm122.2018.1261 
Ceballos, G., A. Szekely, A. García, P. Rodríguez y F. Noguera. 1999. Programa de Manejo de la Reserva de la Biosfera ChamelaCuixmala. Instituto Nacional de Ecología, Secretaría del Medio Ambiente y Recursos Naturales. México, D.F., México. $141 \mathrm{pp}$.

CONANP. 2016. Programa de Manejo Área de Protección de Flora y Fauna la porción norte y la franja costera oriental, terrestres y marinas de la Isla de Cozumel. Comisión Nacional de Áreas Naturales Protegidas-Secetaría de Medio Ambiente y Recursos Naturales. México, D.F., México. 243 pp.

Cruz, K. y V. Cortez. 2015. Hypoxylon (Xylariaceae, Ascomycota) from Western Paraná, Brazil. Brazilian Journal of Botany 38(4): 889-901. DOI: https://doi.org/10.1007/s40415-015-0189-z

Esqueda, M., M. Coronado, A. Gutiérrez, R. Valenzuela, S. Chacón, R. L. Gilbertson, T. Herrera, M. Lizárraga, G. Moreno, E. PérezSilva y T. R. Van Devender. 2010. Hongos. In: Molina-Freaner, F. E. y T. R. Van Devender (eds.). Diversidad Biológica de Sonora. Universidad Nacional Autónoma de México-Comisión Nacional para el Conocimiento y Uso de la Biodiversidad. México, D.F., México. Pp. 189-205, 461-462.

Fournier, J. y C. Lechat. 2015. Three distinctive neotropical species of Hypoxylon (Xylariaceae) with purple $\mathrm{KOH}$-extractable pigments, including two new taxa $H$. cypraeisporum sp. nov. and H. sclerophaeum var. parvisporum var. nov. Ascomycete.org 7 (1): 9-21.

Fournier, J., C. Lechat C. y R. Courtecuisse. 2015. The genus Hypoxylon (Xylariaceae) in Guadeloupe and Martinique (French West Indies). Ascomycete.org 7(5): 145-212.

Guzmán, G. 1983. Los hongos de la Península de Yucatán. II. Nuevas exploraciones y adiciones micológicas. Biotica 8: 71-100.

Guzmán-Dávalos, L., G. Nieves y G. Guzmán. 1983. Hongos del estado de Jalisco II. Boletín de la Sociedad Mexicana de Micología 18: 125-158.

Hernández Sandoval, L. G. 1998. Diversidad florística y endemismo en la Reserva de la Biósfera El Cielo, Tamaulipas, México. Universidad Autónoma de Tamaulipas. Instituto de Ecología Aplicada. Informe final Sistema Nacional de Información sobre Biodiversidad-Comisión Nacional para el Conocimiento y Uso de la Biodiversidad proyecto n. P023. México, D.F., México. $63 \mathrm{pp}$.

Hladki, A. I. y A. I. Romero. 2009. Novedades para los géneros Annulohypoxylon e Hypoxylon (Ascomycota, Xylariaceae) en la República Argentina. Darwiniana 47(2): 278-288.
Ju, Y., M. y J. D. Rogers. 1996. A revision of the genus Hypoxylon. Mycologia Memoir No. 20., APS Press. Saint Paul, USA. 365 pp.

Kuhnert, E., F. Surup, E. Sir, C. Lambert, K. Hyde, A. Hladki, A. Romero y M. Stadler. 2015. Lenormandins A-G, new azaphilones from Hypoxylon lenormandii and Hypoxylon jaklitschii sp. nov., recognised by chemotaxonomic data. Fungal Diversity 71: 165-184. DOI: https://doi.org/10.1007/ s13225-014-0318-1

Martin, P. 1969. Studies in the Xylariaceae V. Euhypoxylon. Journal of South African Botany 35: 149-206.

Medel, R. 2002. Nuevos registros de Pyrenomycetes (Ascomycotina) en México. Boletin de la Sociedad Botánica de México 70: 79-85. DOI: https://doi.org/10.17129/botsci.1656

Medel, R., S. Chacón y G. Guzmán. 1989. Especies conocidas y nuevos registros de Hypoxylon (Sphaeriales, Xylariaceae) en México. Revista Mexicana de Micología 5: 149-168.

Medel, R., G. Guzmán y S. Chacón. 1999. Especies de macromicetos citadas de México IX. Ascomycetes, Parte III: 19831996. Acta Botanica Mexicana 46: 57-72. DOI: https://doi. org/10.21829/abm46.1999.816

Miller, J. H. 1961. A monograph of the world species of HypoxyIon. University of Georgia Press. Athens, USA. 158 pp.

Pérez-Silva, E. 1983. Distribución de algunas especies del género Hypoxylon (Pyrenomycetes) en México. Anales del Instituto de Biología, Universidad Nacional Autónoma de México, Serie Botánica 54: 1-22.

Pérez-Silva, E., M. Esqueda-Valle y A. Armenta-Calderón. 1996. Ascomycetes de Sonora I: Discomycetes y Pyrenomycetes. Revista Mexicana de Micología 12: 97-106.

Petrini, L. y O. Petrini. 1985. Xylariaceous fungi as endophytes. Sydowia 38: 216-234.

Raymundo, T., M. Coronado, A. Gutiérrez, M. Esqueda y R. Valenzuela. 2017. New records of Ascomycota from tropical dry forest in Sonora, Mexico. Mycotaxon 132(2): 421-432. DOI: https://doi.org/10.5248/132.421

Raymundo, T., E. Escudero, I. Ortega, D. Castro-Bustos, H. H. León y R. Valenzuela. 2014. Ascomicetos del bosque tropical caducifolio en el Parque Nacional Lagunas de Chacahua, Oaxaca, México. Boletín de la Sociedad Micológica de Madrid 38: 9-21.

Raymundo, T., R. Soto-Agudelo, S. Bautista-Hernández, A. R. Morales-Campos y R. Valenzuela. 2016. Catálogo de los as- 
comicetos del bosque mesófilo de montaña de Tlanchinol, Hidalgo. Boletín de la Sociedad Micológica de Madrid 40: 83-108.

Raymundo, T., R. Valenzuela, Y. García-Martínez, M. A. BravoÁlvarez, J. C. Ramírez-Martínez, S. Bautista-Hernández, M. Palacios-Pacheco e I. Luna-Vega. 2019. Ascomycetes (fungi) from the relic forest of Fagus grandifolia subsp. mexicana in eastern Mexico. Phytotaxa 418(1): 01-41. DOI: https://doi. org/10.11646/phytotaxa.418.1.1

Rogers, J. D. y Y. M. Ju. 2012. The Xylariaceae of the Hawaiian Islands. North American Fungi 7(9): 1-35. DOI: http://doi. org/10.2509/naf2012.007.009

Rogers, J. D., L. Vasilyeva y F. Hay. 2008. New Xylariaceae from Hawaii and Texas (USA). Sydowia 60 (2): 277-286.

Salinas-Salgado, E., R. Valenzuela, T. Raymundo, M. Cipriano-Salazar, B. Cruz-Lagunas y E. Hernández-Castro. 2012. Macromicetos xilófagos del bosque tropical caducifolio en el municipio de Cocula, Guerrero, México. Polibotánica 34: 209-228.

Sánchez-Jácome, M. R. y L. Guzmán-Dávalos. 2005. New records of ascomycetes from Jalisco, Mexico. Mycotaxon 92: 177-191.

Sánchez-Jácome, M. R. y L. Guzmán-Dávalos. 2011. Hongos citados para Jalisco, II. Ibugana 16: 25-60.

San Martín, F., Ju Y. M. y Rogers J. D. 1999a. Algunas especies de Hypoxylon (Pyrenomycetes, Xylariaceae) de México. Acta Botanica Mexicana 47: 31-53. DOI: https://doi. org/10.21829/abm47.1999.823

San Martín, F., P. Lavín y D. Portugal-Portugal. 1999b. Two new species of Hypoxylon from México. Mycotaxon 72: 159-162.
San Martín, F., J. D. Rogers, P. Lavín, E. Pérez-Silva y M. Esqueda. 1999c. New records of Xylariaceae of Sonora, Mexico. Mycotaxon 71: 129-134.

SEMARNAT. 2015. Área de Protección de Flora y Fauna Silvestres y Acuáticas Sierra de Álamos y Río Cuchujaqui. Comisión Nacional de Áreas Naturales Protegidas-Secretaría de Medio Ambiente y Recursos Naturales. México, D.F., México. $609 \mathrm{pp}$.

Ulloa, M. y R. T. Hanlin. 2006. Nuevo diccionario ilustrado de Micología. APS Press. St. Paul, USA. 615 pp.

Van der Gucht, K., Y. Ju y J. D. Rogers. 1997. New Hypoxylon species from Papua New Guinea and notes on some other taxa. Mycologia 89(3): 503-511. DOI: https://doi.org/10.1080/00 275514.1997.12026809

Vasilyeva, L., S. Stephenson, K. Hyde y A. Bahkali. 2012. Some stromatic pyrenomycetous fungi from northern Thailand-1. Biscogniuxia, Camillea and Hypoxylon (Xylariaceae). Fungal Diversity 55: 65-76. DOI: https://doi.org/10.1007/s13225011-0150-9

Wendt, L., B. Sir, E. Kuhnert, E. Heitkamper, C. Lambert, A. Hladki, A. Romero, J. Luangsa-ard, P. Srikitikulchai, D. Peršoh y M. Stadler. 2018. Resurrection and emendation of the Hypoxylaceae, recognised from a multigene phylogeny of the Xylariales. Mycological Progress 17: 115-154. DOI: https://doi. org/10.1007/s11557-017-1311-3 